\title{
Global optimization method of tool axis vector based on the minimum angular acceleration of rotary feed axis
}

\author{
${ }^{1}$ Jian-wei Ma*, ${ }^{1}$ Guo-qing Hu, ${ }^{1}$ Feng-ze Qin, ${ }^{1}$ Guan-lin Li, ${ }^{1} \mathrm{Zi}$-wen Qu, ${ }^{2}$ Yuanchang Liu \\ ${ }^{1}$ Key Laboratory for Precision and Non-traditional Machining Technology of the Ministry of \\ Education, School of Mechanical Engineering, Dalian University of Technology, Dalian 116024, \\ China. ${ }^{2}$ Department of Mechanical Engineering, University College London, Torrington Place, London \\ WC1E 7JE, UK.

\begin{abstract}
*Corresponding author
Corresponding author: Jian-wei Ma
\end{abstract} \\ Key Laboratory for Precision and Non-traditional Machining Technology of the Ministry of Education, \\ School of Mechanical Engineering, Dalian University of Technology, Dalian 116024, China \\ Telephone: $+86-(0) 411-84707876$ \\ Fax: +86-(0)411-84707743 \\ E-mail address: mjw2011@dlut.edu.cn
}

\begin{abstract}
Complex surface parts are widely used in the industrial applications, and 5-axis NC machining with ballend cutter is the commonly adopted method for curved surface parts. In order to achieve high-quality surface machining, it is especially important to optimize the pose of the tool and the workpiece. The local optimization method of the tool axis vector can effectively improve the machining quality of the curved surface parts with abrupt curvature. However, when the tool axis vector optimizing interval is overmuch, the local optimization method of tool axis vector is time-consuming and ineffective. In order to solve this problem, a global optimization method of tool axis vector based on the minimum angular acceleration is proposed in this study. Firstly, the feasible spaces of tool axis vectors at the cutter contact points are obtained. Then, the toolpath is divided by the property of concavity or convexity for the toolpath curve, and the key tool axis vectors on the toolpath curve are determined. Finally, the tool axis vectors are optimized based on the minimum angular acceleration of rotary feed axis in each interval, and the tool axis vectors at the joint position of the adjacent segments are adjusted to smooth tool axis vectors for the entire toolpath. Simulation and experiment on a test part are carried out to verify the validity of the proposed method, and the global optimization method can effectively improve the machining quality of the complex surface and reduce the machining errors.
\end{abstract}

Key words: Curved surface; Tool axis vector; Global optimization; Rotary feed axis; Kinematical parameters

\section{Introduction}

Complex curved parts are widely used in aerospace, shipbuilding, automobile and other fields. Since most of the complex curved surfaces are applied in the field of high-end equipment, so the shape design of the curved surface is closer to integration, and the machining precision is also higher and higher. In order to meet the machining requirements of complex curved surface with high precision, the traditional 3 -axis machining is difficult to meet the requirements due to the limited posture between the cutter and 
the workpiece. So, the 5-axis machine tools are widely used in machining complex curved surfaces because of the more flexible tool movement space. Compared with the 3-axis NC machine tool, 5-axis NC machine tool has two additional rotary freedoms, making the control of the tool axis direction more difficult during machining. The tool axis vector can be adjusted by tilt angles and yaw angles of the cutter relative to the workpiece, so that the cutting relationship between the cutter and the workpiece can be ensured, and the machining quality can be improved. However, in the process of complex curved surface machining, the machining quality is often affected by the unreasonable tool axis vectors. Especially the curvature of the curved surface changes greatly, the angle between adjacent tool axis vectors is often too large. When the feed rate is large, the angular velocity of the rotary feed axis increases sharply, which easily exceeds the constraints of the machine tool, affecting the smoothness of the machining and causing vibration marks on the machined surface.

In order to avoid the above problems, it can be solved in two aspects. The most common method is to reduce the cutter feed rate, so the kinematical parameters (the angular velocity and the angular acceleration) of the rotary feed axis will decrease, but this will affect the machining efficiency. Another method is to further optimize the tool axis vectors based on the toolpath planning, which will not affect the processing efficiency. In our previous research, a local optimization method for the blade surface with a sharp curvature is given. The tool axis vector optimization is performed by selecting the tool axis vector optimizing interval. However, when the tool axis vector optimizing interval is overmuch, the proposed local optimization algorithm is difficult to guarantee the optimization effectiveness for the tool axis vector. Aiming at the above problem, this study proposes a global optimization method of the tool axis vector based on the minimum angular acceleration for the rotary feed axis, which can effectively solve the phenomenon of overmuch tool axis vectors optimizing intervals and provide guarantee for the high precision machining of complex surface. The research of tool axis vectors optimization method based on the kinematical characteristics is also a hot and difficult point in the 5-axis NC machining.

There are lots of researches on the planning of tool axis vector in the 5-axis NC machining. In order to avoid the interference between tools and parts, Ahmed et al. [1] proposed a new and efficient method of interference detection and avoidance by the shortest cutter. Li et al. [2] proposed a novel generation method of interference-free inspection path for the impeller blades using an on-machine probe. Tang et al. [3] proposed a sweep plane algorithm for the global collision detection with workpiece geometry update for 5-axis NC machining. Morishige et al. [4] proposed a two-dimensional configuration space algorithm to avoid the interference and collision of the cutter axis vector. Jung et al. [5] proposed a 5axis machining toolpath generation method for complex curved surface with high distortion. Wang et al. [6] proposed a general collision detection and automatic tool pose adjustment method for multi-axis milling to eliminate the local interference. Wang et al. [7] proposed a tool interference checking method based on the principle of spatial three-dimensional coordinate system transformation to improve the detection efficiency and the numerical control processing efficiency. In order to generate toolpath meeting the demands of high-speed machining, Sun et al. [8] proposed a direct spiral toolpath generation method with constant scallop-height. Chiou et al. [9] presents a machining potential field (MPF) method to generate toolpaths for the multi-axis sculptured surface machining. Srijuntongsiri et al. [10] proposed and analyzed a new numerical algorithm to reduce the kinematic errors of a 5-axis toolpath by using minimisation of the variation of the rotation angles.

In order to optimize the tool axis vector, Tang et al. [11] proposed a five-axis spiral milling method for tool vector control in the five-axis laser additive manufacturing. Tunc et al. [12] proposed a process simulation integrated tool axis selection approach to adjust the tool axis vectors in an already generated 
5-axis milling path for the improved process stability. Zhu et al. [13] presented an optimization method to select a tool orientation for machining a sculptured surface by the $3+2$-axis machining strategy. Lin et al. [14] researched the irregular machined surface textures appearing in the process of avoiding five-axis singularities using the $\mathrm{C}$-space based on tool orientation translation method. In order to keep generated toolpaths smooth and continuous, Xu et al. [15] presented a five-axis tool positioning algorithm based on smooth toolpaths. According to the five axis NC machining of ball end milling, Liu et al. [16] presented a new method to optimize tool axis vector based on graph. In order to overcome the large incoherent movement of the rotary feed axis, Ma et al. [17] proposed an optimization method of tool axis vector based on the kinematical characteristics of the rotary feed axis for curved surface machining. Based on the improvement of the C-space method, Zhou et al. [18] proposed a safe space method that could generate continuous and uniform tool axis vectors before and after the interference region. Jun [19] and Tang [20] based on machining surface error analysis and considering the geometry of adjacent parts and the feasible tool direction in $\mathrm{C}$ space, minimized the change of tool direction during machining. Ho et al. [21] proposed a tool orientation smoothing method (TOS method) combined with a cutting error improvement method (CEI method) to reduce cutting errors and improve machining efficiency.

Based on the above, the researches focus on reducing the angle between the tool axis vectors between adjacent cutter contact points as the optimization target. And the kinematic parameters of machine tools, such as angular velocity and angular acceleration of rotary feed axis, are rarely considered. Therefore, it is urgent to carry out the research for the smoothing of the tool axis vector based on the kinematics of the rotary feed axis. Since any direction of the ball-end cutter does not affect the contact state between the cutter and the workpiece, it is widely used in complex curved surface machining. In order to make the kinematics of the rotary feed axis meet the constraints of the machine tool, a global optimization method based on the minimum angular acceleration of the tool axis vector is proposed in this study. With the minimum angular acceleration as the optimization objective, the tool axis vectors are optimized to reduce the angular acceleration of the rotary feed axis without changing the number of cutter contact points, and the machining quality of the complex curved surface with sharp curvature is improved. Firstly, the interference judgment principle is used to calculate the feasible spaces of the tool axis vectors at the cutter contact points. Then, the property of concavity or convexity for the toolpath curve is judged, so as to divide the interval of toolpath curve and the key tool axis vectors on the toolpath curve are determined. Finally, the tool axis vectors on the toolpath are optimized based on the minimum angular acceleration in each interval. On this basis, the tool axis vectors at the joint position of the adjacent intervals are adjusted to ensure smoothing of the tool axis vectors on the entire toolpath. Simulation analysis and experiment on a test parts are implemented to verify the validity of the proposed tool axis vector optimization method for improving the quality complex curved surface parts.

The rest of this paper is organized as following. In Section 2, the kinematical parameters of the rotary feed axis in machine tool is calculated. Global optimization method of tool axis vector based on the minimum angular acceleration is proposed in Section 3. The experiment results and the discussions are in Section 4. Conclusions are summarized in Section 5.

\section{Kinematical characteristics analysis of rotary feed axis in machine tool}

In this section, the toolpath generation method is first given and then the coordinate system transformation is introduced. On this basis, the calculation method of angular velocity and angular acceleration for the rotary feed axis is given, which lay the basis for the optimization of the tool axis vector in curved surface machining. 


\subsection{Toolpath calculation in five-axis machining}

During complex curved surface machining, the machining curved surface can be represented by vectors as

$$
\mathbf{S}(u, v)=[x(u, v), y(u, v), z(u, v)]^{T}
$$

Where $u$ and $v$ are variables of the parametric surface $\mathbf{S}(u, v)$. In general, $u$ and $v$ have a value range of $[0,1]$.

For a given surface $\mathbf{S}(u, v)$, the unit normal vector $\mathbf{N}$ of any point can be expressed as

$$
\mathbf{N}(u, v)=\frac{\mathbf{S}_{u}(u, v) \times \mathbf{S}_{v}(u, v)}{\left\|\mathbf{S}_{u}(u, v) \times \mathbf{S}_{v}(u, v)\right\|}
$$

In which, $\mathbf{S}_{u}(u, v)$ and $\mathbf{S}_{v}(u, v)$ are the first derivatives of the surface.

On the curved surface, the toolpath $\mathbf{r}(\xi)$ can be represented by a parameter curve as

$$
\mathbf{r}(\xi)=[x(\xi), y(\xi), z(\xi)]^{T}
$$

In which, $\xi$ represents the curve parameter, the value range is $[0,1]$. And the unit tangent vector $\mathbf{T}$ can be calculated as

$$
\mathbf{T}=\frac{\mathbf{r}^{\prime}(\xi)}{\left|\mathbf{r}^{\prime}(\xi)\right|}
$$

In the machining process of the curved surface, the cutter contact point $\mathbf{P}_{\mathbf{C}}$ between the cutter and the curved surface is as shown in Fig. 1. $\mathbf{V}$ is the unit tool axis vector and $\mathbf{K}=\mathbf{N} \times \mathbf{T}$ is the cross product of unit normal vector $\mathbf{N}$ and unit tangent vector $\mathbf{T}$ at the cutter contact point. The local coordinate system $P_{C} X_{L} Y_{L} Z_{L}$ is established by setting the $\mathbf{T}, \mathbf{K}$ and $\mathbf{N}$ as the directions of $X_{L}, Y_{L}$ and $Z_{L}$ at the cutter contact point $\mathbf{P}_{\mathbf{C}}$. For ball-end cutter, the coordinates relationship between the cutter tip point $\mathbf{P}$ and the cutter contact point $\mathbf{P}_{\mathbf{C}}$ is obtained as

$$
\mathbf{P}_{C}=\mathbf{P}+R \cdot \mathbf{V}-R \cdot \mathbf{N}
$$

In which, $R$ is the radius of the ball-end cutter.

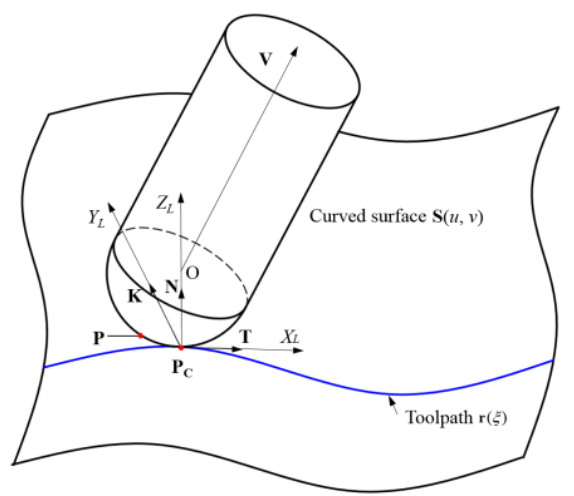

Fig. 1 Machining surface and local coordinate system.

In this study, the toolpaths of cutter contact points in the machining process are generated according to the equal residual scallop height method. The important parameters in the toolpath generation algorithm are the side-step length and the step-size. And the side-step length $L$ is the distance between the corresponding cutter contact points of the adjacent toolpaths, which is related to the scallop height $h$. The step-size $L_{S}$ refers to the distance between adjacent cutter contact points on the same toolpath, which is related to the chord height error $\varepsilon$ [22]. The curved surface machining schematic shown in Fig. 2 provides a good understanding of the concept of side-step length and step-size. 


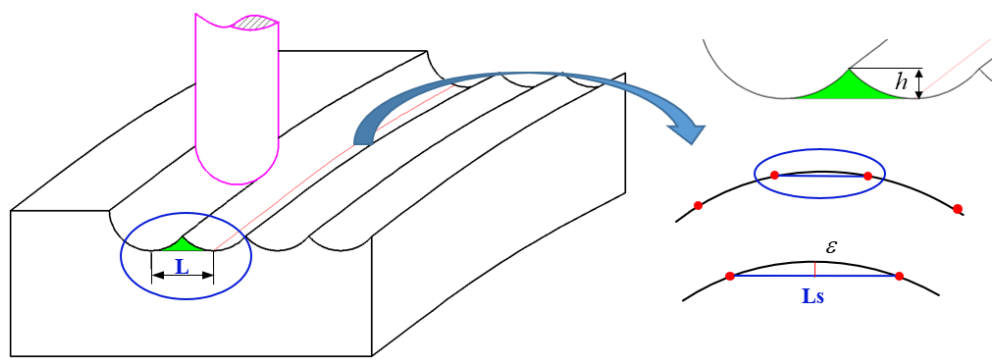

Fig. 2 Surface machining schematic diagram.

In order to calculate the side-step length, it is necessary to distinguish the surface features according to gaussian curvature and mean curvature. For a plane as shown in Fig. 3, when the maximum residual scallop height $h$ is given, the corresponding side-step length $L$ can be obtained as

$$
L=2 \sqrt{R^{2}-(R-h)^{2}}=2 \sqrt{2 R h-h^{2}}
$$

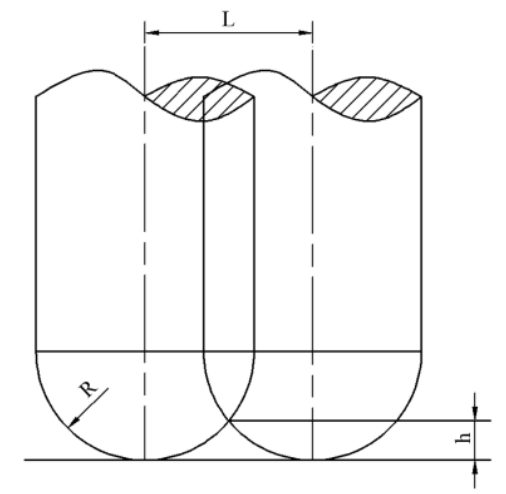

Fig. 3 Calculation schematic of side-step length for plane.

For a convex surface, assuming the radius of curvature is $\rho$ as shown in Fig. 4, the calculation equation for side-step length is as follows

$$
h=\frac{(\rho+R)}{\rho} \sqrt{\rho^{2}-\left(\frac{L}{2}\right)^{2}}-\sqrt{R^{2}-\left[\frac{\rho+R}{\rho} \frac{L}{2}\right]^{2}}-\rho
$$

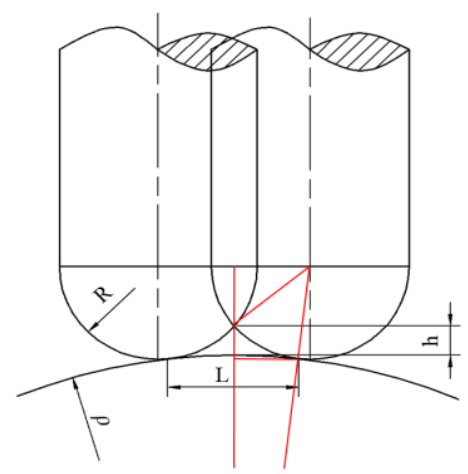

Fig. 4 Calculation schematic of side-step length for convex surface.

In general, the residual scallop height $h<<\rho$, so the Eq. (7) is simplified to

$$
L=\sqrt{8 h R\left(\frac{\rho}{R+\rho}\right)^{2}}=2 \sqrt{2 h R} \frac{\rho}{(\rho+R)}
$$

For a concave surface as shown in Fig. 5, the side-step length can be obtained as

$$
L \approx 2 \sqrt{2 h R} \frac{\rho}{(\rho-R)}
$$




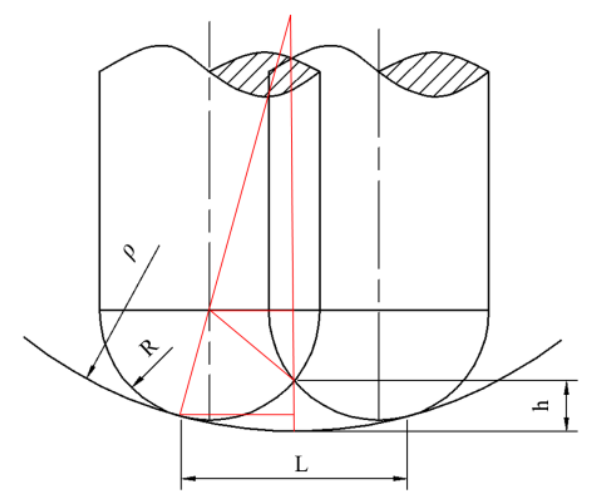

Fig. 5 Calculation schematic of side-step length for convex surface.

As shown in Fig. 6, the straight segments are used to approximate the ideal trajectory in the actual machining. In order to control the quality of the machining surface, a reasonable chord height error is set in the actual machining. After giving the chord height error, the step-size can be calculated as

$$
L_{s}=2 \sqrt{\rho^{2}-(\rho-\varepsilon)^{2}}=2 \sqrt{2 \rho \varepsilon-\varepsilon^{2}}
$$

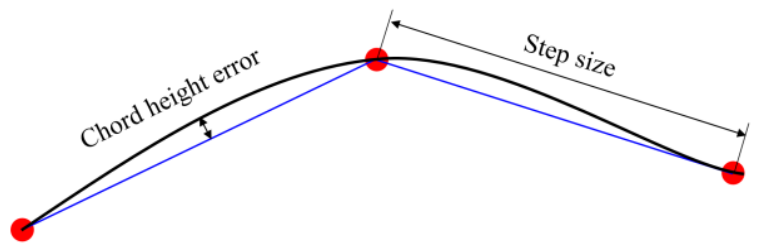

Fig. 6 Model of step-size.

The toolpath in the five-axis machining not only includes the cutter tip point trajectory, but also the pose of the cutter during the movement, that is the tool axis vector [23]. As shown in the Fig. 7, the angle $\alpha$ along the $Y_{L}$-axis is defined as tilt angle and the angle $\beta$ along the $Z_{L}$-axis is defined as yaw angle in the local coordinate system. According to the unit normal vector, the tool axis vectors in local coordinate system expressed by tilt angles $\alpha$ and yaw angles $\beta$ can be obtained as

$$
\mathbf{V}_{\text {axis }}^{L C S}=\boldsymbol{\operatorname { R o t }}(\mathbf{N}, \beta) \cdot \boldsymbol{\operatorname { R o t }}(\mathbf{K}, \alpha) \cdot[0,0,1,0]^{T}
$$

In which, $\mathbf{V}_{a x i s}^{L C S}$ represents the tool axis vector in the local coordinate system.

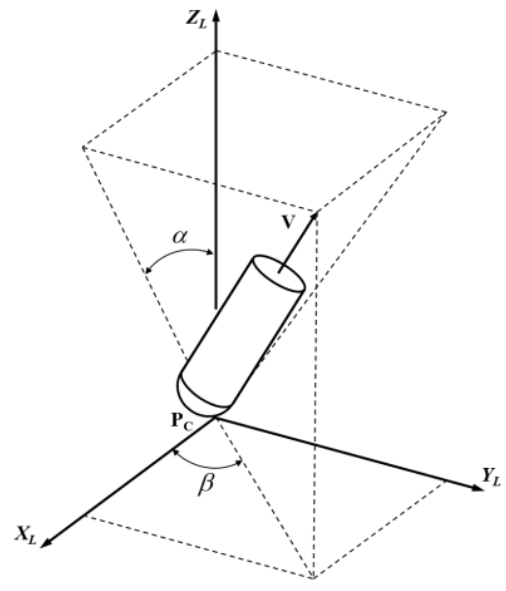

Fig. 7 Tool axis vector in local coordinate system.

From the above, the toolpath calculation method including cutter tip points and tool axis vectors on the surface is obtained.

\subsection{Inverse kinematics of $A C$ rotary axis machine tool}

In 5-axis NC machining, the toolpath can be obtained through the method in the Section 2.1. In general, the cutter location files can be obtained in the CAM software, which contains cutter tip points 
coordinates $\mathbf{P}[x, y, z]^{\mathrm{T}}$ and tool axis vectors $\mathbf{V}\left[i^{*}, j^{*}, k^{*}\right]^{\mathrm{T}}$ expressed in the workpiece coordinate system. However, the cutter location files generated by the CAM software need to be transformed into the machine coordinate system, so as to be recognized by the machine tool with $A C$ rotary axis. Therefore, the transformation between workpiece coordinate system and machine tool coordinate system needs to be established.

The tool axis vectors $\mathbf{V}=\left[i^{*} j^{*} k^{*} 0\right]^{\mathrm{T}}$ in the workpiece coordinate system can be obtained by rotating the vector $\mathbf{V}_{\mathbf{M}}=\left[\begin{array}{llll}0 & 0 & 1 & 0\end{array}\right]^{\mathrm{T}}$ in the machine coordinate system around $A$-axis and $C$-axis. The equation can be obtained as

$$
\mathbf{V}=\boldsymbol{\operatorname { R o t }}\left(\mathbf{Z}, \theta_{C}\right) \cdot \boldsymbol{\operatorname { R o t }}\left(\mathbf{X}, \theta_{A}\right) \cdot \mathbf{V}_{M}
$$

In which, $\theta_{A}$ and $\theta_{C}$ represent the rotary angles of $A$-axis and $C$-axis respectively, and the relationship between the workpiece coordinate system and machine coordinate system is obtainend as

$$
\begin{aligned}
\mathbf{M} & =\boldsymbol{\operatorname { R o t }}\left(\mathbf{Z}, \theta_{C}\right) \cdot \boldsymbol{\operatorname { R o t }}\left(\mathbf{X}, \theta_{A}\right) \\
& =\left[\begin{array}{cccc}
\cos \theta_{C} & -\cos \theta_{A} \sin \theta_{C} & \sin \theta_{A} \sin \theta_{C} & 0 \\
\sin \theta_{C} & \cos \theta_{A} \cos \theta_{C} & -\sin \theta_{A} \cos \theta_{C} & 0 \\
0 & \sin \theta_{A} & \cos \theta_{A} & 0 \\
0 & 0 & 0 & 1
\end{array}\right]
\end{aligned}
$$

Therefore, from the Eq. (12) and Eq. (13), the tool axis vector in the workpiece coordinate system is obtained as

$$
\left\{\begin{array}{l}
i^{*}=\sin \theta_{A} \cdot \sin \theta_{C} \\
j^{*}=-\sin \theta_{A} \cdot \cos \theta_{C} \\
k^{*}=\cos \theta_{A}
\end{array}\right.
$$

Thus the inverse kinematics conversion relation of the tool axis vectors between the machine coordinate system and the workpiece coordinate system are established as

$$
\left\{\begin{array}{l}
\theta_{A}=\arctan 2\left(\sqrt{i^{* 2}+j^{* 2}}, k^{*}\right) \\
\theta_{C}=\arctan 2\left(i^{*}, j^{*}\right)
\end{array}\right.
$$

According to the above, the relationships between the tool axis vectors in workpiece coordinate system and machine coordinate system are established. So as to obtain the $\mathrm{G}$ code that can be identified by machine tool in the machine coordinate system.

\subsection{Calculation of angular velocity and angular acceleration for rotary feed axis}

For the machining surface, the toolpath of machining can be obtained by Section 2.1. During machining, the ball-end cutter contacts the curved surface at the cutter contact points and cuts along the toolpath. And the tool axis vectors are guaranteed to move along the specified pose. If the toolpath is complex, especially with abrupt curvature, it is easy to cause the angular velocity and the angular acceleration for the rotary feed axis change too much, which affects the machining quality.

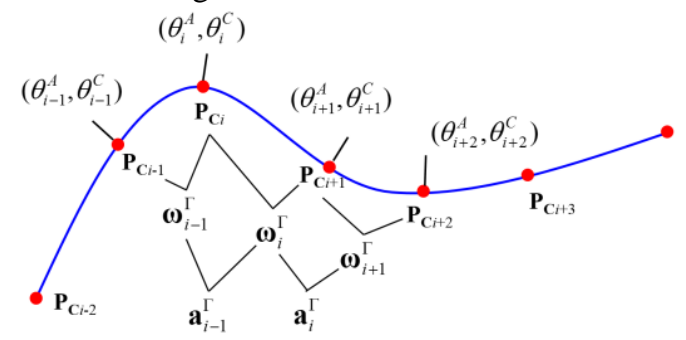

Fig. 8 Calculation diagram of angular velocity and angular acceleration of rotary feed axis.

In order to reduce the kinematical parameters, the angular velocity and the angular acceleration of the rotary feed axis are calculated by the discrete method. The toolpath obtained in Section 2.1 contains $n$ cutter contact points and $\mathbf{P}_{\mathbf{i}}$ is the $i$ th cutter contact point. At any cutter contact point $\mathbf{P}_{\mathbf{i}}$, the cutter 
location files can be expressed as $\{\mathbf{P}, \mathbf{V}\}=\left\{x, y, z, i^{*}, j^{*}, k^{*}\right\}$. It is assumed that the feed speed is $v$ when the cutter moves from the cutter contact point $\mathbf{P}_{\mathbf{C}_{i}}$ to $\mathbf{P}_{\mathbf{C}_{i+1}}$, the approximate arc length between the cutter contact points $\mathbf{P}_{\mathbf{C}_{i}}$ and $\mathbf{P}_{\mathbf{C}_{i+1}}$ is $L_{i}$, and the tool axis vector changes from $\mathbf{V}_{i}$ to $\mathbf{V}_{i+1}$. According to the inverse kinematics of the machine tool in Section 2.2, the $A$-axis and $C$-axis angle values of the machine tool can be obtained at the corresponding cutter contact posits, just changing from $\theta_{A}$ and $\theta_{C}$ to $\theta_{A+1}$ and $\theta_{C+1}$ respectively. As shown in the Fig. 8, for discrete points on the toolpath, the angular velocity and the angular acceleration of the rotary feed axis can be solved as

$$
\left\{\begin{array}{l}
\boldsymbol{\omega}_{i}^{\Gamma}=v \cdot \frac{\theta_{i+1}^{\Gamma}-\theta_{i}^{\Gamma}}{L_{i}} \\
\mathbf{a}_{i}^{\Gamma}=2 v \cdot \frac{\boldsymbol{\omega}_{i+1}^{\Gamma}-\boldsymbol{\omega}_{i}^{\Gamma}}{L_{i+1}+L_{i}}
\end{array} \quad \Gamma=A \text { or } C\right.
$$

In which, $\theta_{i}^{\Gamma}$ represent the rotation angles of $A$-axis and $C$-axis in the machine tool corresponding for the $i$ th cutter contact point. $\boldsymbol{\omega}_{i}^{\Gamma}$ and $\mathbf{a}_{i}^{\Gamma}$ represent the angular velocity and the angle acceleration of $A$-axis and $C$-axis in the machine tool corresponding for the $i$ th cutter contact point.

Thus, the synthetic angular velocity and the angular acceleration for rotary feed axis can be calculated as

$$
\begin{gathered}
\boldsymbol{\omega}_{i}= \begin{cases}\frac{\theta_{i+1}-\theta_{i}}{L_{i}} \cdot v=\sqrt{\boldsymbol{\omega}_{A i}{ }^{2}+\boldsymbol{\omega}_{C i}{ }^{2}} & i=1, \ldots, n-1 \\
0 & i=n\end{cases} \\
\mathbf{a}_{i}= \begin{cases}2 v \cdot \frac{\boldsymbol{\omega}_{i+1}-\boldsymbol{\omega}_{i}}{T_{i+1}+T_{i}}=\sqrt{\mathbf{a}_{A i}{ }^{2}+\mathbf{a}_{C i}{ }^{2}} & i=2, \ldots, n-1 \\
0 & i=1, n\end{cases}
\end{gathered}
$$

Through the above analysis, the kinematical parameters of the rotary feed axis of the machine tool is recognized, so the angular velocity and the angular acceleration for rotary feed axis at any cutter contact point can be obtained.

\section{Global optimization of tool axis vector based on minimum angular acceleration}

In Section 2, the transforming relationship of tool axis vector between workpiece coordinate system and the machine coordinate system is obtained. Then, the calculation method of kinematical parameters for the rotary feed axis of the machine tool is given. In this section, a global optimization method for tool axis vector is given.

In the process of local optimization of the tool axis vector, when the tool axis vector optimizing interval is overmuch, the local optimization method of tool axis vector is time-consuming and ineffective. In order to solve this problem effectively, a global optimization method of tool axis vector based on the minimum angular acceleration is proposed. In the global optimization of the tool axis vector, there is a large amount of calculation when optimizing the tool axis vector of the entire toolpath directly, which is easy to produce a solution-free phenomenon. In order to reduce the calculation amount effectively and avoid the problem that the tool axis vector has no solution, the idea of global optimization is proposed based on segmented toolpath. Firstly, according to the interference judgment criterion, the feasible spaces of the tool axis vectors in the local coordinate system are calculated. Then, the toolpath curve is segmented and the key tool axis vectors are set by the property of concavity or convexity for the toolpath curve. Finally, based on the principle of the minimum angular acceleration of the rotary feed axis, an improved tool axis vector interpolation algorithm is used to optimize the tool axis vectors in each interval. 
On this basis, the tool axis vectors at the joint position of the adjacent intervals are adjusted to ensure the smoothing of tool axis vectors on the entire toolpath.

\subsection{Solving of feasible spaces for tool axis vectors}

In the process of tool axis vectors optimization, a common method is to optimize the tool axis vectors firstly and then judge whether the interference occurs between the cutter and the workpiece. When the curved surface is mostly convex, this method is more practical. However, there is interference between the cutter and the workpiece in most cases, so the tool axis vector needs to be adjusted again. In order to effectively avoid the interference occurs to cutters and workpieces, the feasible space solution method of tool axis vector for each cutter contact point is given. And the feasible spaces of cutter contact points are solved, which lays a foundation for the global optimization method of tool axis vectors.

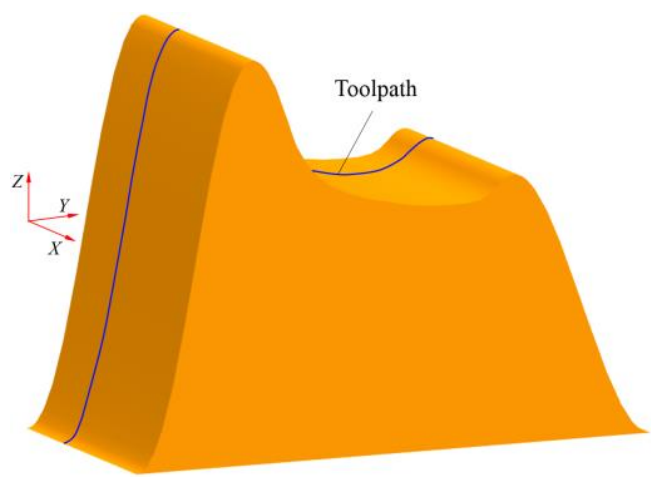

Fig. 9 Curved surface and toolpath.

In the global optimization of the tool axis vector, the machining toolpath is first generated by the equal residual scallop height method according to the shape of the surface. As shown in the Fig. 9 in the $\mathrm{UG}, N$ toolpaths are generated by direction-parallel cutting, each of which has $n_{k}$ cutter contact points $(k=1, \ldots, N)$. This study takes one of the toolpath as an example to illustrate the global optimization process of the tool axis vector. The interference between the cutter and the workpiece is a common phenomenon in the five-axis machining. In order to avoid interference after the tool axis vector optimization, according to the geometric characteristics of the surface, the C-space method is used to solve the feasible space of the tool axis vector in the local coordinate system of each cutter contact point on the toolpath. In terms of local interference, in order to prevent the cutter from interfering with the workpiece, the effective cutting radius of the cutter should be smaller than the minimum radius of curvature at the cutter contact point, so the local interference-free region can be obtained as

$$
\rho_{t}<\rho_{S \min }
$$

In which, $\rho_{t}$ represents the effective cutting radius of the tool. For the ball-end cutter, the effective cutting radius is the cutter radius, $\rho_{S \min }$ represents the minimum curvature radius of the machined surface.

In terms of global interference, in order to avoid the collision between the cutter and the workpiece, the cutter is simplified into a cylinder with an infinite length, the surface of the cutter is $\mathbf{S}^{t}$, and the surface to be machined is $\mathbf{S}^{r}$. The global interference-free region can be obtained as

$$
d_{r, t}=\left[\mathbf{S}^{t}-\mathbf{S}^{r}\right] \cdot \mathbf{N}>0
$$

Where $d_{r, t}$ represents the directed distance between the machined surface $\mathbf{S}^{r}$ and the cutter surface $\mathbf{S}^{t}$, and $\mathbf{N}$ represents the normal direction of the surface at certain cutter contact point.

In order to quickly solve the feasible space of the tool axis vector in the local coordinate system, the $\mathrm{C}$-space method is used in this study to traverse the tilt angle $\alpha$ and the yaw angle $\beta$. The two-dimensional $\mathrm{C}$-space coordinate system is established as shown in the Fig. 10 by taking the tilt angle $\alpha$ as the vertical 
axis and the yaw angle $\beta$ as the horizontal axis. In this coordinate system, a rectangular region composed of $\alpha \epsilon[-\pi / 2, \pi / 2]$ and $\beta \in[-\pi, \pi]$ is called a $\mathrm{C}$-space. Any point $(\alpha, \beta)$ of the $\mathrm{C}$-space can represent the tool axis vector pose with a tilt angle of $\alpha$ and a yaw angle of $\beta$ in the local coordinate system. In order to calculate the feasible tool axis vector in C-space, the two-dimensional C-space is discreted to obtain the matrix grid of $M \times N$ columns. By judging whether each matrix grid satisfies the Eq. (19) and Eq. (20), the feasible space of the tool axis vector in the local coordinate system is obtained. In the traversal process, the coordinates of the four vertices of each rectangular grid can be expressed as $\left[\left(\alpha_{i}, \beta_{j}\right),\left(\alpha_{i+1}, \beta_{j}\right),\left(\alpha_{i+1}, \beta_{j+1}\right),\left(\alpha_{i}, \beta_{j+1}\right)\right]$. In order to make the calculation simplify, the intermediate point is used to represent the tool axis vector pose, so it can be expressed as

$$
C_{i, j}=\left[\left(\alpha_{i}+\alpha_{i+1}\right) / 2,\left(\beta_{j}+\beta_{j+1}\right) / 2\right]
$$

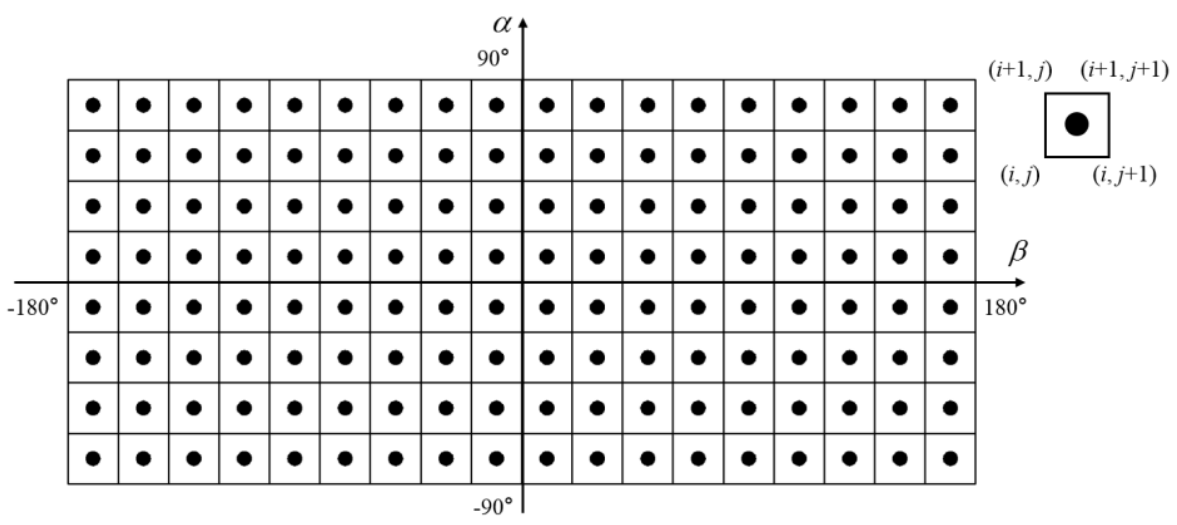

(a) C space gridding

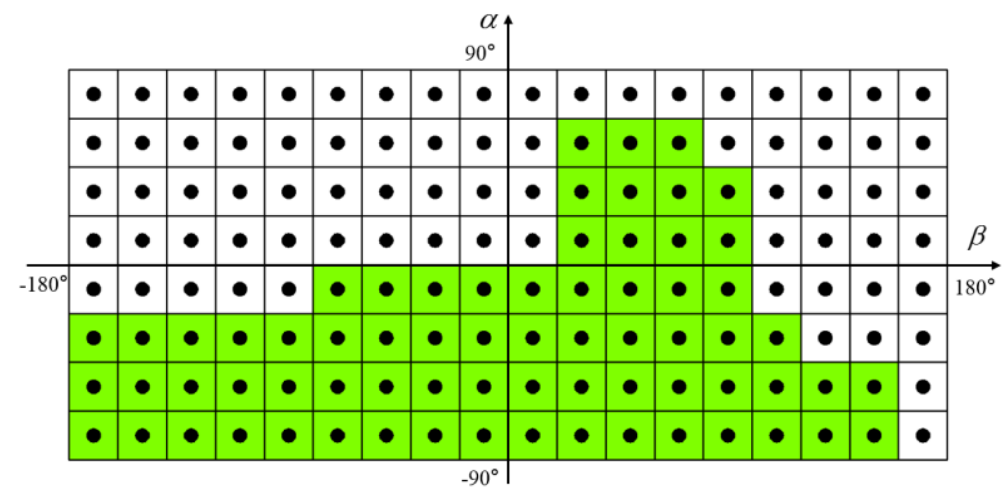

(b) Feasible space of tool axis vector

Fig. 10 Discrete solution of feasible space for tool axis.

The feasible space of the tool axis vector at each cutter contact point representing by the tilt angle and yaw angle in the local coordinate system can be obtained by the $\mathrm{C}$ space method. According to the transformation relationship between the local coordinate system and the workpiece coordinate in Section 2.2 , the feasible spaces of tool axis vectors in workpiece coordinate system are obtained, and then the feasible spaces of the tool axis vectors indicated by the rotary angles $A$ and $C$ in the machine coordinate system can be obtained by inverse kinematics transform.

Through the above method, the feasible space of the tool axis vector at the cutter contact point in different coordinate systems can be obtained, and the constraint conditions for tool axis vector optimization are determined, which lays a foundation for the global optimization of the tool axis vector.

\subsection{Key cutter contact points selection and tool axis vector determination}

In Section 3.1, the feasible space of the tool axis vector at each cutter contact point in different coordinate systems can be obtained, which provides constraints for tool axis vector optimization. There 
are a large number of computational defects when directly optimize the tool axis vector on the entire toolpath curve. In order to reduce the problem of long calculation time due to large calculation amount, the toolpath needs to be segmented. For a toolpath curve, there are convex curve and concave curve. Therefore, according to the principle of concavity and convexity, the toolpath curve is divided into multiple curve segments, and the key tool axis vectors are set at the end position of each curve segment, and then the tool axis vectors of each interval segment are optimized to achieve the overall optimization. However, for a given toolpath, it is generally difficult to express the toolpath information using equations. Therefore, it is necessary to obtain the concavity and convexity of the toolpath curve by discrete method and divide the toolpath curve according to the property of concavity or convexity for the toolpath. The end point and the junction of concavity and convexity of the toolpath, that is the inflection point, are used as the key points of the tool axis vector. Constrained by the feasible spaces of the cutter contact points, the tool axis vector is optimized as the critical tool axis vector, and the tool axis vectors in the interval are homogenized to obtain the smoothing tool axis vector without interference on the entire toolpath.

For a given curve, the curve equation is $f(x)$ and the curve is assumed to be monotonic in the interval $\left[x_{1}, x_{2}\right]$. The concavity and convexity of the curve can be judged by the endpoint value and the midpoint value, as shown in Fig. 11.

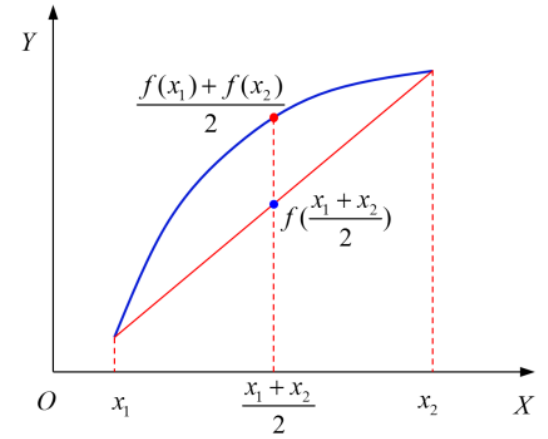

(a) Convex curve

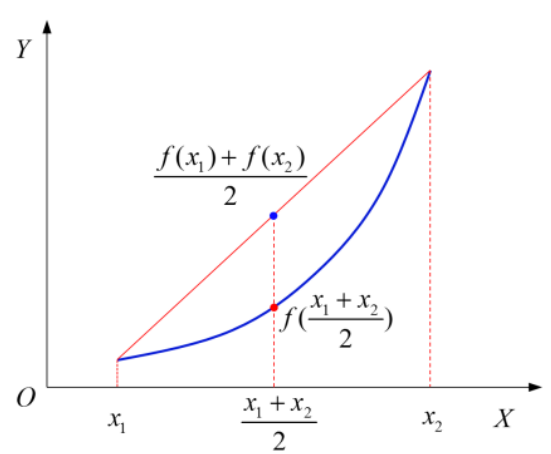

(b) Concave curve

Fig. 11 Judgment of concavity and convexity.

As shown in the Fig. 11(a), according to the relationship between the curve and the line in the figure, it can be seen that the curve in the figure satisfies the following equation as

$$
\begin{gathered}
f\left(\frac{x_{1}+x_{2}}{2}\right)>\frac{f\left(x_{1}\right)+f\left(x_{2}\right)}{2} \\
f^{\prime \prime}(x)<0
\end{gathered}
$$

When the curve equation satisfies the Eq. (22) and Eq. (23), the curve is a convex curve. Similarly, the curve in the Fig. 11(b) satisfies the equation as

$$
\begin{gathered}
f\left(\frac{x_{1}+x_{2}}{2}\right)<\frac{f\left(x_{1}\right)+f\left(x_{2}\right)}{2} \\
f^{\prime \prime}(x)>0
\end{gathered}
$$

When the curve equation satisfies the Eq. (24) and Eq. (25), the curve is a concave curve.

However, for a curved surface, the machining toolpath curve cannot be clearly expressed by the equation most of the time. In this case, the cutter location files of the toolpath curve obtained by UG are used to judge the concavity and convexity of the curve by means of discrete methods. The cutter location files contain the cutter position information $\left\{\{\mathbf{P}, \mathbf{V}\}_{1},\{\mathbf{P}, \mathbf{V}\}_{2}, \ldots,\{\mathbf{P}, \mathbf{V}\}_{\mathrm{n}}\right\}$ of the $n$ cutter contact points on the curve. From the calculation method of the cutter contact points described in the Section 2.1, the coordinates of the cutter contact points $\mathbf{P}_{\mathrm{C}_{\mathrm{i}}}=\left(x c_{i}, y c_{i}, z c_{i}\right)$ on the toolpath curve can be obtained. Using the finite difference method to solve the derivative, the first derivative of toolpath curve can be expressed as 


$$
f^{\prime}\left(x_{i, i+1}\right)=\frac{z c_{i+1}-z c_{i}}{\sqrt{\left(x c_{i+1}-x c_{i}\right)^{2}+\left(y c_{i+1}-y c_{i}\right)^{2}}} \quad 1 \leq i \leq n-1
$$

The second derivative of the toolpath curve is as

$$
f^{\prime \prime}\left(x_{i}\right)=\frac{z c_{i+1}-2 z c_{i}+z c_{i-1}}{\sqrt{\left(x c_{i}-x c_{i-1}\right)^{2}+\left(y c_{i}-y c_{i-1}\right)^{2}} \sqrt{\left(x c_{i+1}-x c_{i}\right)^{2}+\left(y c_{i+1}-y c_{i}\right)^{2}}} \quad 2 \leq i \leq n-1
$$

The first and second derivatives on the toolpath curve can be calculated from Eq. (26) and Eq. (27). The point where the first derivative of the toolpath curve is zero is called the extreme point. According to the value of the second derivative of the toolpath curve, concavity and convexity of the toolpath curve can be judged. When the second derivative satisfies the formula $f^{\prime \prime}\left(x_{i}\right)>0$, the curve is a concave curve, and when the formula $f^{\prime \prime}\left(x_{i}\right)<0$, the curve is a convex curve. After solving the concavity and convexity, the point that satisfies the formula $f^{\prime \prime}\left(x_{i}\right)=0$ is called the inflection point, which is the boundary point of the toolpath curve. After calculation, the sequence number of the cutter contact points of the extreme point and the inflection points on the toolpath can be obtained. Thus, the position of the extreme points and the inflection points on the toolpath curve can be obtained, as shown in Fig. 12.

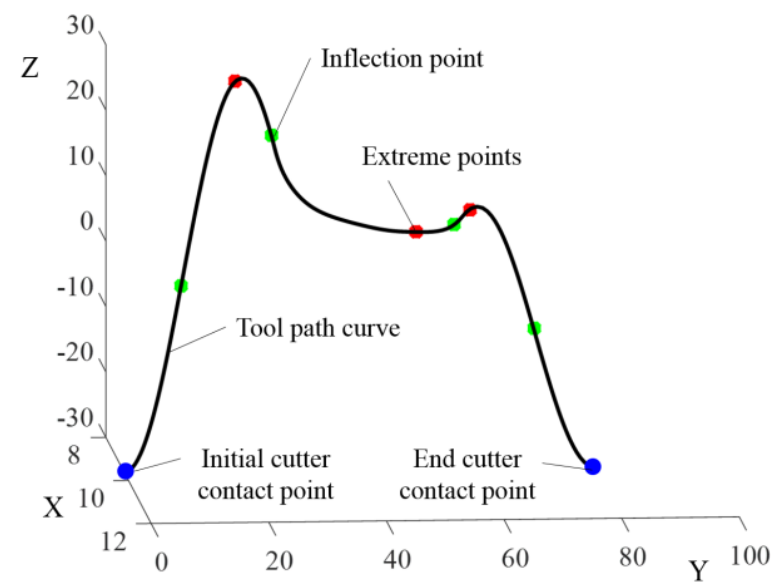

Fig. 12 Position of extreme point and inflection point on toolpath curve.

Suppose there are $m$ inflection points on a toolpath, and the key tool axis vectors are set at the inflection points of the toolpath as the boundary. The toolpath curve can be divided into $m+1$ interval segments $\left[e_{i}, f_{i}\right]$. Constrained by the feasible spaces of the critical tool axis vectors, the optimal direction of the key tool axis is set, and the tool axis vectors are optimized in each interval segment to achieve the global optimization of the tool axis vector.

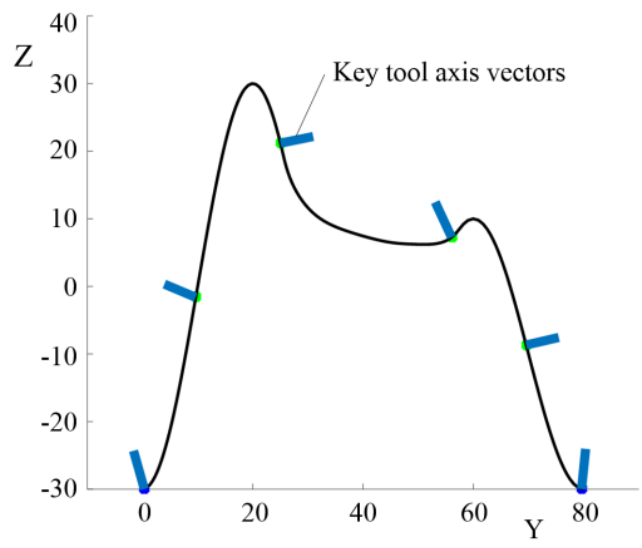

Fig. 13 Key tool axis vector selection.

The position of the key cutter contact points on the toolpath can be found by the method mentioned above, as shown in Fig. 13. According to the feasible space solution method in Section 3.1, the feasible 
spaces for the tool axis vectors of the cutter contact points can be obtained. With this constraint, by adjusting the tool axis vector at this position, it is ensured that the tool axis vector smooth transition during the machining process, reducing the vibration caused by the unsteady feed of the machine tool rotary feed axis, and improving the surface quality.

\subsection{Tool axis vector optimization and adjustment}

In Section 3.1, the solution method of the feasible spaces of the tool axis vectors at the cutter contact points are given. In Section 3.2, the tool axis vectors $\mathbf{V}\left[\mathbf{V}_{\text {fir }}, \mathbf{V}_{1}, \ldots, \mathbf{V}_{\mathrm{m}}, \mathbf{V}_{\text {end }}\right]$ at the key cutter contact points are obtained through the judgment of concavity and convexity of the toolpath curve, and the toolpath curve is divided into $m+1$ interval segments, which lays a foundation for the global optimization method of the tool axis vector. In this section, the tool axis vector optimization method in each interval is mainly introduced.

In order to better express the optimization effect of tool axis vector, the objective function of the tool axis vector is constructed as

$$
\left\{\begin{array}{l}
\min f=\sum_{i=1}^{n-1}\left(\omega_{i+1}-\omega_{i}\right)^{2} \\
\text { s.t. } \quad \mathbf{V}_{i} \in \mathbf{\Omega}_{i}
\end{array}\right.
$$

In which, $\omega_{i}$ is the angular velocity value at the $i$ th cutter contact point, $\mathbf{V}_{i}$ is the tool axis vector at the $i$ th cutter contact point, and $\boldsymbol{\Omega}_{i}$ is the feasible space of the tool axis vector at the $i$ th cutter contact point.

In order to get the minimum value of the function, the Eq. (28) is derived to obtain the Eq. (29) as

$$
f^{\prime}=2 \sum_{i=1}^{n-1}\left(\omega_{i+1}-\omega_{i}\right)
$$

When $\omega_{i+1}=\omega_{i}$, the above formula takes the minimum value.

The feed rate cannot be kept constant during the actual machining process. In this study, the effect of the feed rate is ignored and the feed rate between adjacent cutter contact points are considered constant. The following relationship exists between adjacent cutter contact points in surface machining.

$$
\begin{aligned}
& \omega_{i} t_{i}=\theta_{i} \\
& L_{i}=v \cdot t_{i}
\end{aligned}
$$

Where $\theta_{i}$ is the angle between the tool axis vectors of adjacent cutter contact points, $L_{i}$ is the arc length between adjacent cutter contact points, $v$ is the tool feed speed, and $t_{i}$ is the time the tool passes the adjacent cutter contact points.

Combine the Eq. (30) and Eq. (31), it can be obtained as

$$
\theta_{i}=\omega_{i} \cdot \frac{L_{i}}{v}
$$

When the feed rate $v$ is considered to be constant, the minimum value of the objective function occurs at $\omega_{i+1}=\omega_{i}$, so there is

$$
\frac{\theta_{i}}{\theta_{i+1}}=\frac{L_{i}}{L_{i+1}}
$$

According to the relationship of Eq. (33), it can be obtained as

$$
\frac{\theta_{i}}{\theta_{i}+\theta_{i+1}}=\frac{L_{i}}{L_{i}+L_{i+1}}
$$




$$
\frac{\sum_{i=e}^{i} \theta_{i}}{\sum_{i=e}^{f} \theta_{i}}=\frac{\sum_{i=e}^{i} L_{i}}{\sum_{i=e}^{f} L_{i}}
$$

From the above derivation, in order to achieve the minimum angular acceleration of the rotary feed axis, the angle of the tool axis vector between adjacent cutter contact points should be proportional to the arc length between adjacent cutter contact points. Furthermore, it can be seen that the proportion of the angle of the adjacent tool axis vector to the total tool axis vector in the optimizing interval $[e, f]$ should be equal to the proportion of the arc length of the adjacent cutter contact points in the total arc length.

In Section 3.2, $m+1$ toolpath intervals and $m$ key tool axis vectors are obtained. One of the intervals $[e, f]$ is taken as an example to illustrate the tool axis vector optimization method. The tool axis vector at the cutter contact point $\mathbf{P}_{\mathbf{C} e}$ is $\mathbf{V}_{e}$, and the tool axis vector at the cutter contact point $\mathbf{P}_{\mathbf{C} f}$ is $\mathbf{V}_{f}$. The tool axis vector in the interval can be calculated by formula interpolation.

$$
\mathbf{V}_{i}=\kappa(\lambda) \cdot \mathbf{V}_{e}+\mu(\lambda) \cdot \mathbf{V}_{f} \quad \lambda \in[0,1]
$$

In which, $\kappa(\lambda)$ and $\mu(\lambda)$ are coefficients about parameter variable $\lambda, \lambda \in[0,1]$.

In order to avoid the problem of uneven angle of the adjacent tool axis vectors caused by linear interpolation, the spherical interpolation method is adopted, as shown in the Fig. 14.

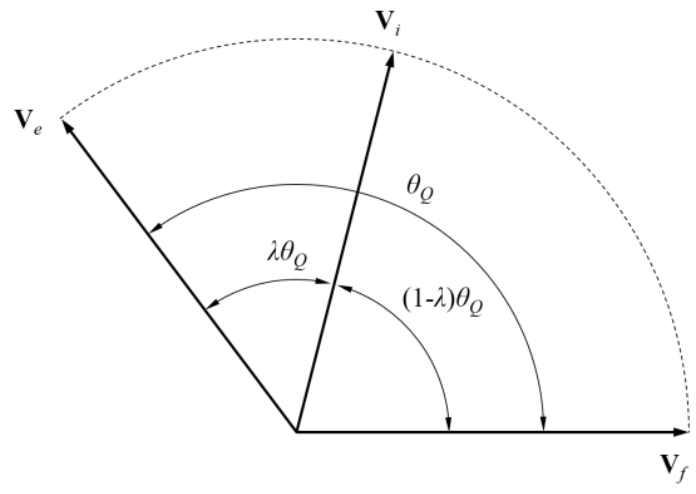

Fig. 14 Spherical interpolation method.

In order to determine the coefficients, Eq. (36) should do dot product with $\mathbf{V}_{e}$ and $\mathbf{V}_{f}$ respectively and can be expressed by the following formula, as

$$
\left\{\begin{array}{l}
\mathbf{V}_{e} \cdot \mathbf{V}_{i}=\kappa(\lambda) \mathbf{V}_{e} \cdot \mathbf{V}_{e}+\mu(\lambda) \mathbf{V}_{e} \cdot \mathbf{V}_{f} \\
\mathbf{V}_{f} \cdot \mathbf{V}_{i}=\kappa(\lambda) \mathbf{V}_{f} \cdot \mathbf{V}_{e}+\mu(\lambda) \mathbf{V}_{f} \cdot \mathbf{V}_{f}
\end{array}\right.
$$

For $\mathbf{V}_{e}, \mathbf{V}_{f}$, and $\mathbf{V}_{i}$ are all the unit tool axis vector, Eq. (37) can be translated as

$$
\left\{\begin{array}{l}
\cos \lambda \theta_{Q}=\kappa(\lambda)+\mu(\lambda) \cos \theta_{Q} \\
\cos \left[(1-\lambda) \theta_{Q}\right]=\kappa(\lambda) \cos \theta_{Q}+\mu(\lambda)
\end{array}\right.
$$

From the Eq. (38), the coefficient calculation can be obtained as

$$
\left\{\begin{array}{l}
\kappa(\lambda)=\frac{\sin \left(\theta_{Q} \cdot(1-\lambda)\right)}{\sin \theta_{Q}} \\
\mu(\lambda)=\frac{\sin \left(\theta_{Q} \cdot \lambda\right)}{\sin \theta_{Q}}
\end{array}\right.
$$


In order to make the optimization function take the minimum value, the calculations of the variables $\lambda$ and $\theta_{Q}$ are obtained as

$$
\left\{\begin{array}{l}
\lambda=\frac{\sum_{i=e}^{i} L_{i}}{\sum_{i=e}^{f} L_{i}} \\
\theta_{Q}=\arccos \frac{\mathbf{V}_{e} \cdot \mathbf{V}_{f}}{\left|\mathbf{V}_{e} \| \mathbf{V}_{f}\right|}
\end{array}\right.
$$

By Eqs. (36)-(40), the improved calculation method of the tool axis vector considering the distance between adjacent cutter contact points can be derived as

$$
\mathbf{V}_{i}=\frac{\sin \left(\theta_{Q}-\frac{\sum_{i=e}^{i} L_{i}}{\sum_{i=e}^{f} L_{i}} \theta_{Q}\right.}{\sin \theta_{Q}} \mathbf{V}_{e}+\frac{\sin \left(\frac{\sum_{i=e}^{i} L_{i}}{\sum_{i=e}^{f} L_{i}} \theta_{Q}\right.}{\sin \theta_{Q}} \mathbf{V}_{f}, \quad i \in[e+1, f-1]
$$

The Eq. (41) considers the problem of the kinematic fluctuation of the rotary feed axis due to the difference in machining time caused by the unequal arc length between adjacent cutter contact points, so that the kinematics of the rotary feed axis of the machine tool is more stable.

From the above analysis, the optimized tool axis vector in the interval can be obtained by the improved tool axis vector optimization method in each tool axis vector optimizing interval $\left[e_{i}, f_{i}\right]$, and the overall tool axis vector optimization process is shown in Fig. 15.

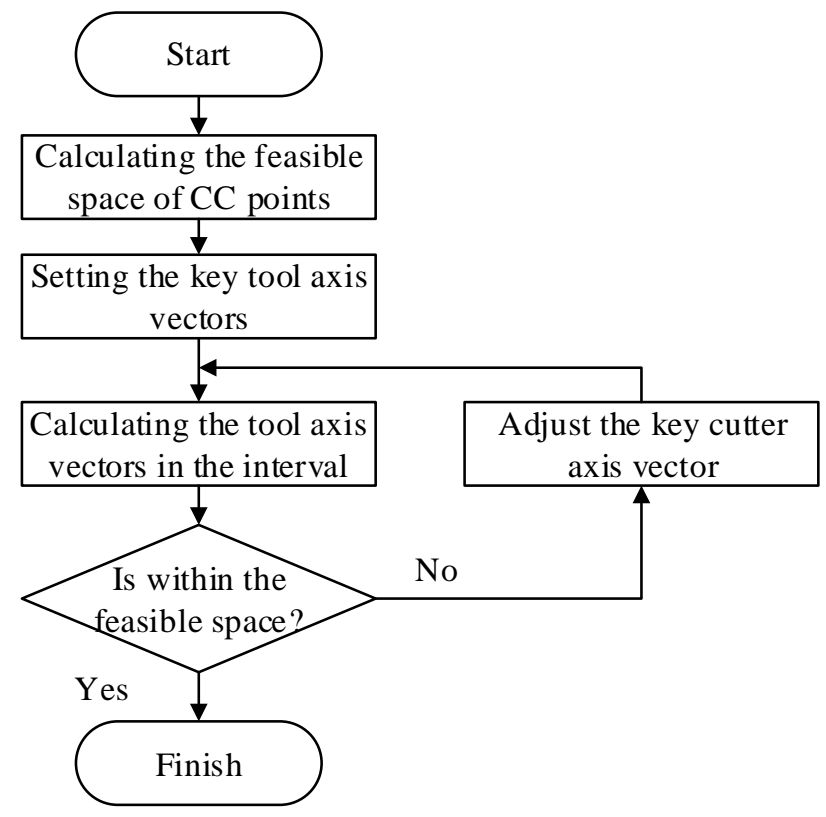

Fig. 15 Tool axis vector optimization flowchart.

When the proposed global optimization method is used directly to optimize the tool axis vectors, it is easy to cause the large tilt angle of the tool axis vector because of the asymmetrical distribution of the convex section of the toolpath, as shown in the Fig. 16, which is not conducive to the machining.

In order to avoid the above issue, when the curvature of toolpath interval changes greatly, the extreme point should be guaranteed to be in the middle of the interval of toolpath. For the position in Fig. 16 , a tool axis vector is inserted in the convex section of the toolpath to make it symmetric, as shown in Fig. 17. And the optimized tool axis vectors are obtained as shown in Fig. 18. 


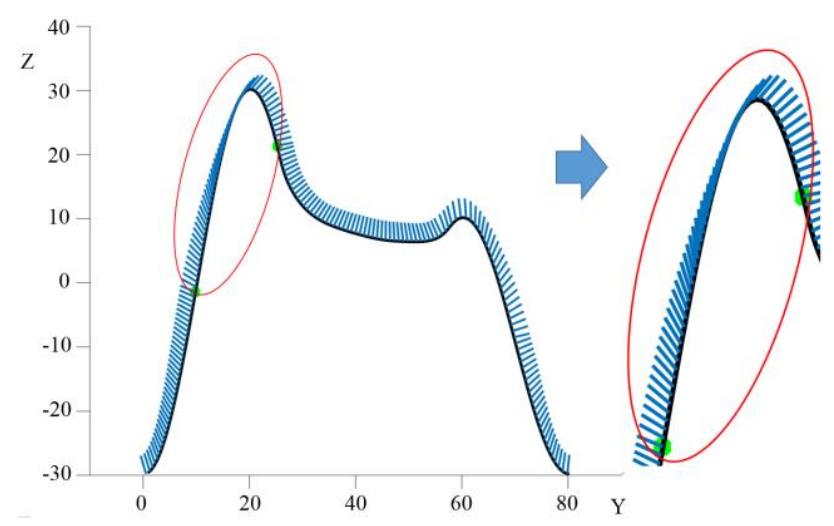

Fig. 16 Large tilt angle of tool axis vector due to asymmetrical distribution of convex section in toolpath.

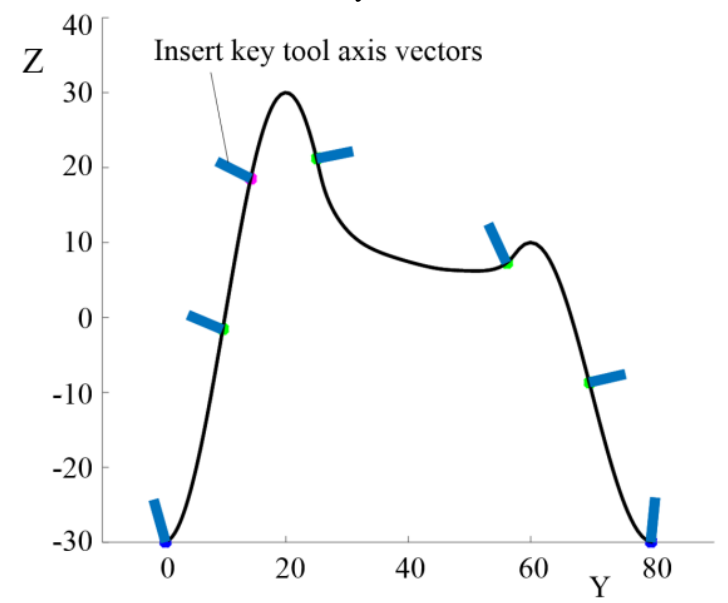

Fig. 17 Division of tool axis vector optimizing interval.

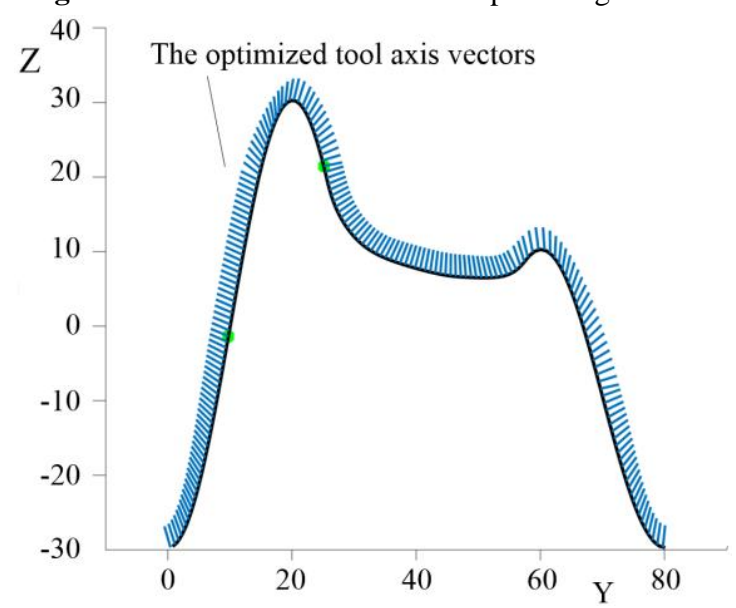

Fig. 18 Optimized tool axis vecters.

Through the above method, the optimized tool axis vectors are obtained in each interval, and the angular acceleration of the rotary feed axis is minimized. However, in the optimization of cutter axis vector, only the tool axis vector smoothing in one interval is considered, and the tool axis vector smoothing between adjacent sections is not considered. In order to avoid the abrupt change of the angular velocity between the adjacent interval segments $\left[e_{i}, f_{i}\right]$ and $\left[e_{i+1}, f_{i+1}\right]$, as shown in Fig. 19, the tool axis vectors in this region are re-optimized by the tool axis homogenization idea to meet the smooth transition of the tool axis vector at adjacent sections, so as to ensure the smooth transition of the tool axis vector on the entire toolpath. 


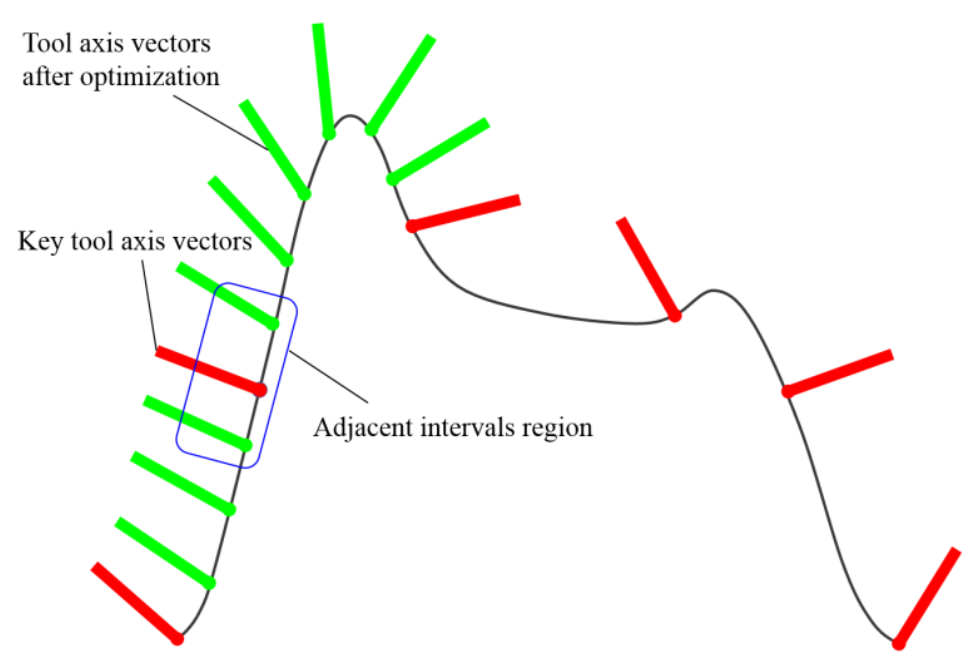

Fig. 19 Schematic diagram of tool axis vector global optimization.

\section{Experiment results and discussion}

In order to further verify the effectiveness of the proposed global optimization method for the tool axis vector, a sine surface is designed for experimental verification. As shown in Fig. 9, the initial cutter position files are obtained after setting the residual height and the step size by using the line cutting method. And the angular velocity and angle acceleration of the rotary feed axis can be calculated by the cutter position files. According to the improved tool axis vector optimization algorithm proposed in Section 3, the effectiveness of the method is verified from both simulation and experimental verification.

\subsection{Simulation analysis}

Commercial CAM software, such as UG, generates a machining toolpath by setting a fixed tilt angle and yaw angle. The global optimization method of the tool axis vector is given in Section 3. In this section, the kinematics of the rotary feed axis of the machine tool is simulated to verify the validity of the proposed method.

For the experimental example a sinusoidal surface is established by UG as shown in Fig. 9, the tilt angle is $5^{\circ}$ and the yaw angle is $0^{\circ}$. In order to simulate accurately, the processing parameters are consistent with the experiment. The spindle speed is set to $3000 \mathrm{r} / \mathrm{min}$, the feed rate is $250 \mathrm{~mm} / \mathrm{min}$, the maximum residual scallop height during machining is set to $0.02 \mathrm{~mm}$, the cutting mode is one-way row cutting, and the SANDVIK two-blade ball-end milling cutter with a diameter of $6 \mathrm{~mm}$ is used for machining. So the cutter location files are obtained, and the angle values of rotary feed axis are obtained as shown in Fig. 20. According to the Sections 3.1 and 3.2, the feasible space of the tool axis vector and the direction of the key tool axis vector can be obtained. The optimized tool axis vector can be obtained by globally optimization method proposed in Section 3.3. Taking the cutter contact points sequences $\{1$ $8\}$ of the toolpath as an example to illustrate the change of tool axis vector before and after optimization. The initial tool axis vectors for the cutter contact points $1-8$ are $\{(0,-03281,0.9447),(0,-0.6063,0.7952)$, $(0,-0.7259,0.6878),(0,-0.7870,0.6169),(0,-0.8267,0.5626),(0,-0.8500,0.5268),(0,-0.8679,0.4968)$, $(0,-0.8804,0.4742)\}$, the corresponding angles of the rotary feed axis of the machine tool are $\{(-19.1507$, 180), (-37.3222, 180), (-46.5477, 180), (-51.9082, 180), (-55.7644, 180), (-58.2102, 180), (-60.2138, $180),(-61.6904,180)\}$. According to the improved tool axis vector optimization method proposed in Section 3.3, the optimized tool axis vectors are $\{(0,-03281,0.9447),(0,-0.3512,0.9363),(0,-0.3737$, 0.9276), (0, -0.3952, 0.9186), (0, -0.4185, 0.9082), (0, -0.4390, 0.8985), (0, -0.4611, 0.8874), (0, -0.4816, $0.8764)\}$, and the corresponding angles of the rotary feed axis are $\{(-19.1507,180),(-20.5602,180),(-$ $21.9411,180),(-23.2801,180),(-24.7359,180),(-26.0394,180),(-27.4562,180),(-28.7914,180)\}$. 

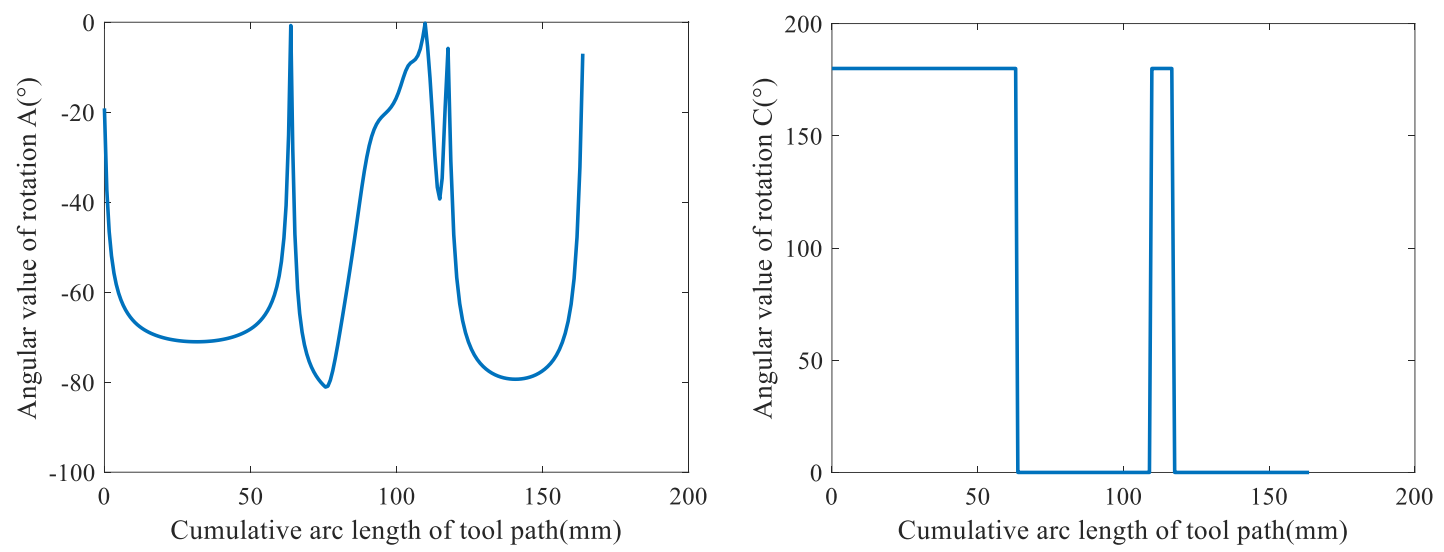

Fig. 20 Relationship between rotation angle of machine rotary feed axis and cumulative arc length of toolpath before tool axis vector optimization.

In order to explain the effectiveness of the tool axis vector optimization from the simulation point of view, the feed speed is set to $250 \mathrm{~mm} / \mathrm{min}$ during the simulation. And the angle coordinate value and the synthetic kinematics parameters (angular velocity and angular acceleration) of the $A C$ rotary feed axis of the machine tool are calculated through Section 2.3 in MATLAB. The cumulative arc length of the toolpath is taken as the horizontal coordinate, and the kinematical parameters of the $A C$ axis are taken as the vertical coordinate, the synthetic angular velocity and angular acceleration of rotary feed axis before tool axis vector optimization are as shown in Fig. 21.
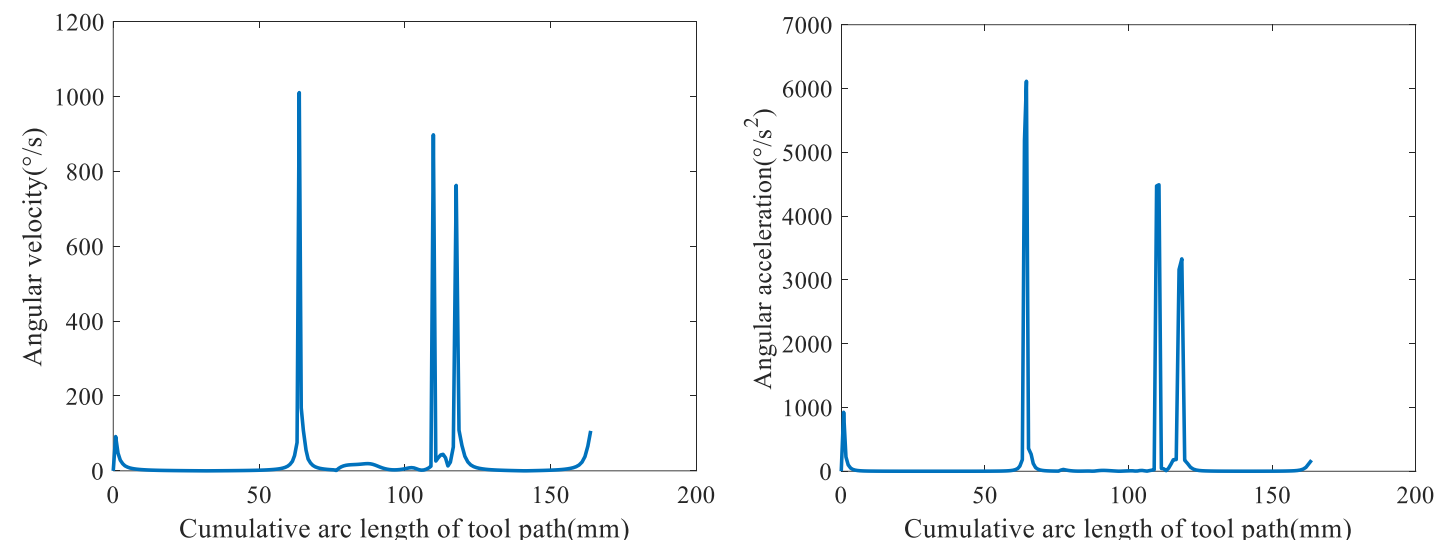

Fig. 21 Synthetic kinematic parameters of rotary feed axis and cumulative arc length of toolpath before tool axis vector optimization.
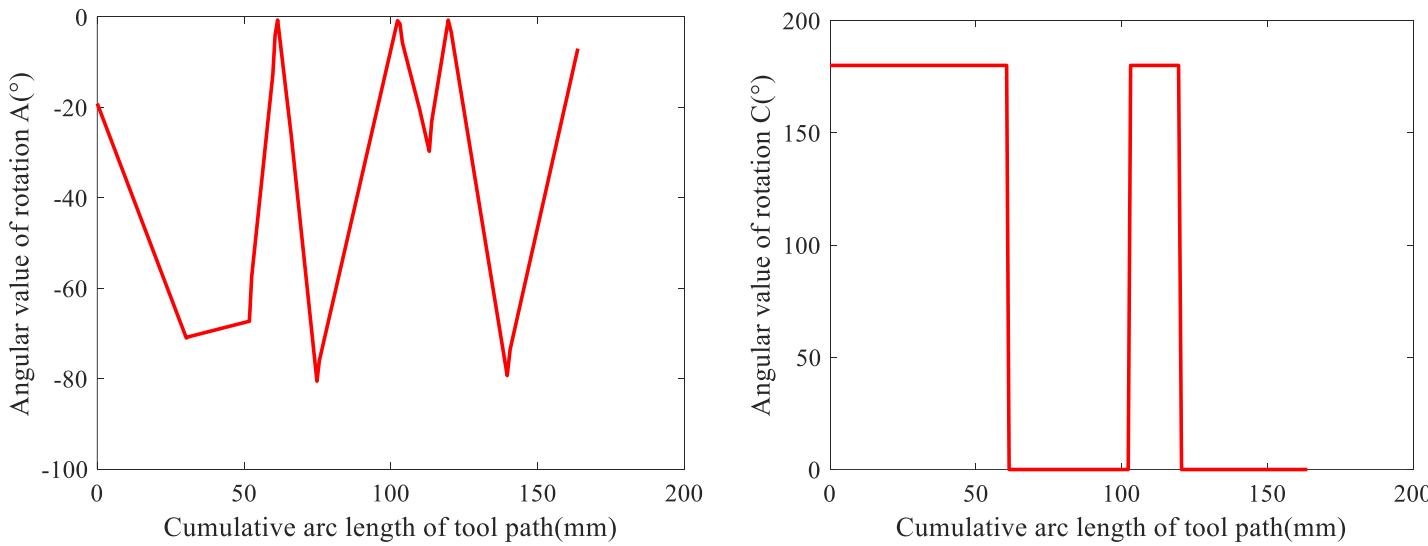

Fig. 22 Relationship between rotation angle of the machine rotary feed axis and cumulative arc length of toolpath after tool axis vector optimization.

In order to make the rotary feed axes of machine tools smoothly, an improved global optimization method based on the minimum angular acceleration is proposed in Section 3. After global optimization 
for the tool axis vectors, the relationship between the angle value of the rotary feed axis of machine tool and the cumulative arc length of the toolpath is shown in Fig. 22. And the relationship between synthetic kinematic parameters of the $A C$ rotary feed axis and the cumulative arc length curve of the toolpath is shown in Fig. 23.
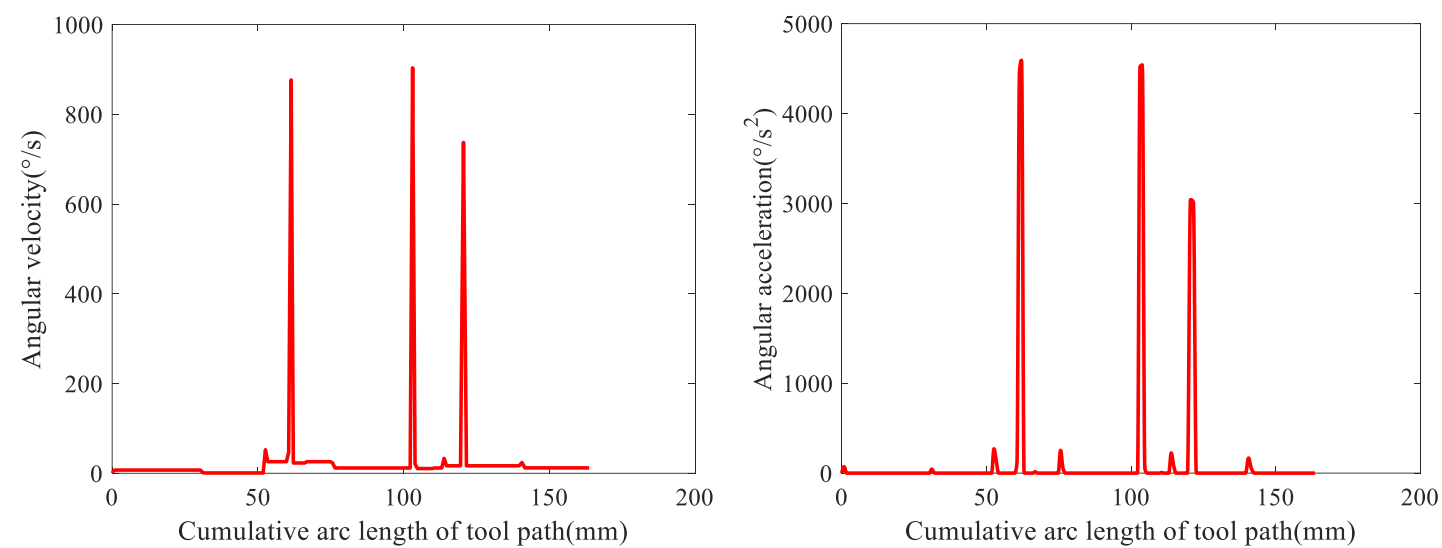

Fig. 23 Synthetic kinematic parameters of rotary feed axis and cumulative arc length of toolpath after tool axis vector optimization.

It can be clearly seen from Fig. 21 and Fig. 23 that there are three large fluctuations in the relationship between the angular velocity and the angular acceleration of the rotary feed axis and the cumulative arc length of the toolpath. The reason for the large fluctuation of this position is that the limitation of the $A$-axis rotation range of the machine tool causes the $C$-axis to produce three times of $180^{\circ}$ large rotations. According to the above simulation, the angular velocity and angular acceleration of the rotary feed axis of machine tool are reduced after the global optimization of the tool axis vector. After the tool axis vector optimization, the maximum angular velocity of the rotary feed axis is reduced from $1010 \%$ s to $900 \%$, which is reduced by $10.9 \%$, and the maximum angular acceleration is reduced from $6110 \% \mathrm{~s}^{2}$ to $4590 \% \mathrm{~s}^{2}$, which is reduced by $24.8 \%$. It can be seen from the simulation analysis that after the tool axis vector optimization, the rotary feed axis of machine tool has better stability, and the angular velocity and angular acceleration fluctuation are also significantly reduced, which theoretically improves the surface quality.

\subsection{Experimental verification}

In order to verify the effectiveness of the optimization method for tool axis vectors, an actual processing experiment is carried out in this chapter. For the sine surface with the sharp-curvature characteristics as shown in Fig. 9, the aviation 7075 aluminum alloy was used as the raw material to carry on the test verification on the five-axis NC milling machine. The processing parameters are consistent with those set in simulation. The machining cutter is shown in Fig. 24.

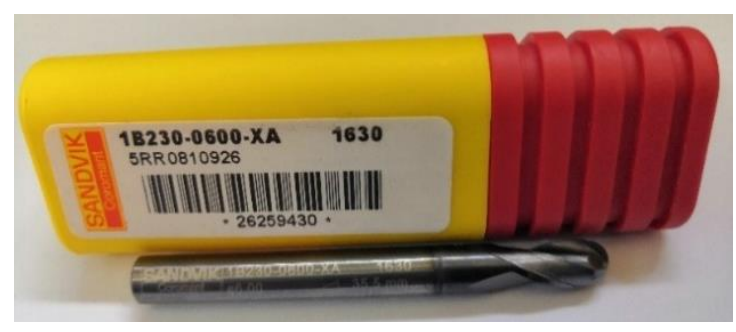

Fig. 24 SANDVIK 1B230-0600-XA 1630 type ball-end milling cutter.

In order to avoid the machining errors caused by positioning and to convenient to compare the machining effects, the global optimization method of the tool axis vector proposed in the study and the traditional method of tool axis vector planning are used for finishing machining on the same rough piece 
in the experiment. The experimental machining process is shown in Fig. 25. As shown in Fig. 26, the machining result for global optimization method of the tool axis vector proposed in this study is compared with that of conventional method for tool axis vector planning.
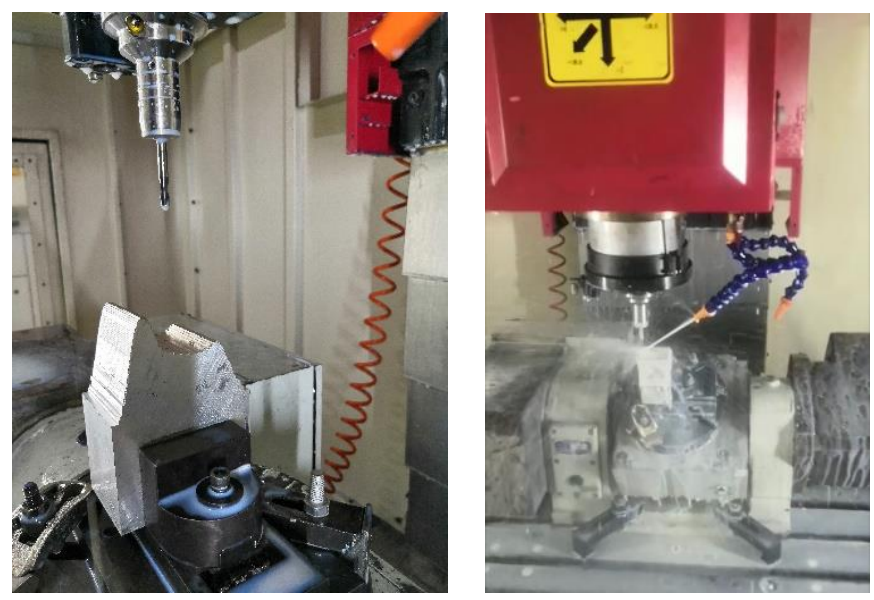

Fig. 25 Experimental machining process of parts with sharp curvature

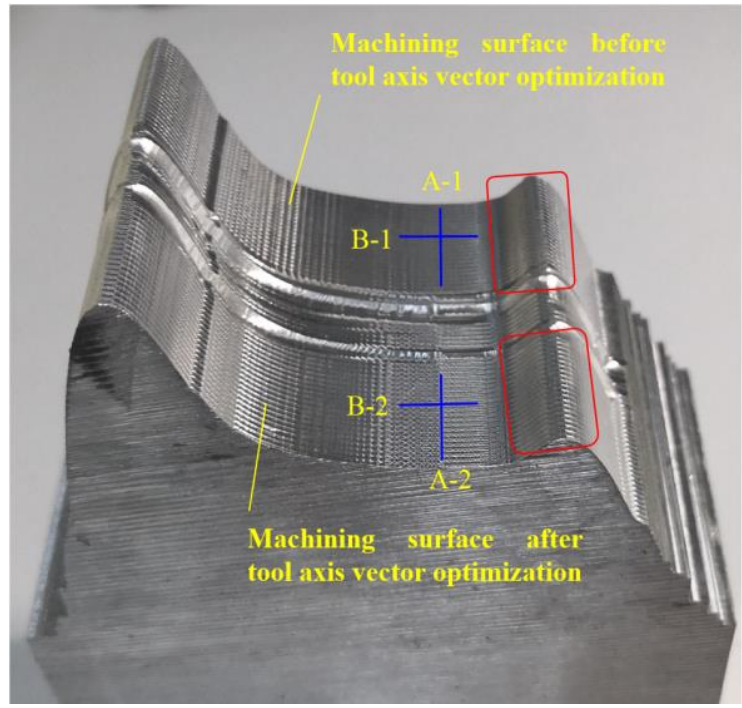

Fig. 26 Machining results before and after tool axis vector optimization

Due to the large change of the angular velocity and the angular acceleration of the rotary feed axis of the machine tool, the kinematical performances of the machine tool are difficult to meet the requirements, so that the machined surface has obvious chatter marks, so as to affect the machining quality. In Section 4.1, it is verified by simulation analysis that the global optimization method based on the minimum angular acceleration of the tool axis vector proposed in this paper can significantly reduce the kinematical parameters of the rotary feed axis of the machine tool, so it is of great significance to improve the machining quality.

In order to verify the effectiveness of the global optimization method based on the minimum angular acceleration of the tool axis vector proposed in this study for improve the machining quality, the contact roughness profiler (PGI 840) was used to measure the surface roughness. As shown in the Fig. 26, the direction $A$ reflects the surface roughness, which is determined by the scallop height between the adjacent toolpaths, so the surface roughness of the $A$ direction unchange before and after the tool axis vector optimization. And the direction of $B$ reflects the machining contour, which can verify the optimization effect for tool axis vector. The measured surface roughness in the $A$ and $B$ direction is shown in Fig. 27 and Fig. 28 respectively. It can be seen from Fig. 27 that the surface machining quality does not change 
substantially along the $A$ direction. As shown in Fig. 28, the surface roughness in the $B$ direction after the global optimization method of the tool axis vector is slightly better than that before the tool axis vector optimization. The main reason is that the curvature of this area is relatively small, and the effect of tool axis vector optimization is relatively small.
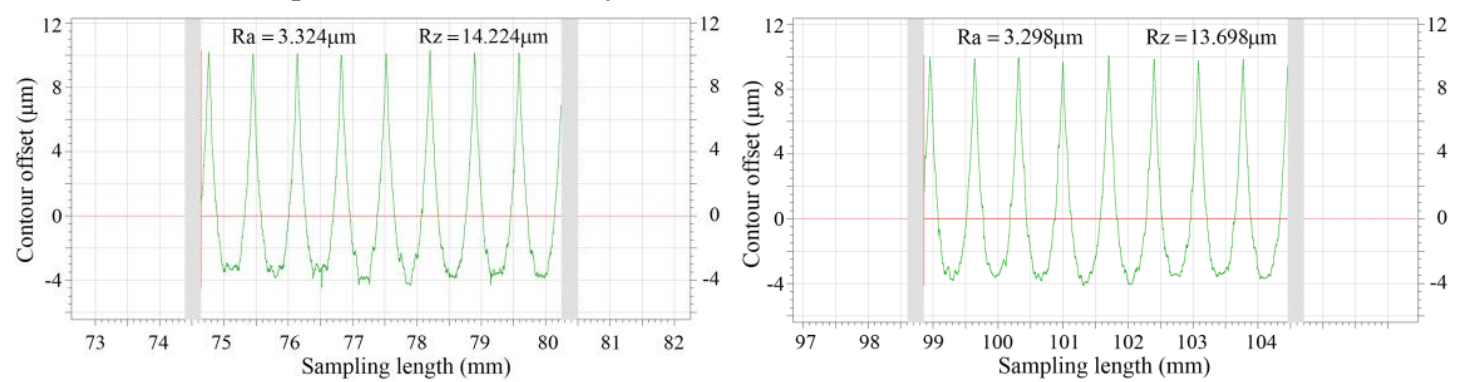

Fig. 27 Surface roughness in direction $A$ before and after optimizing for tool axis vector.
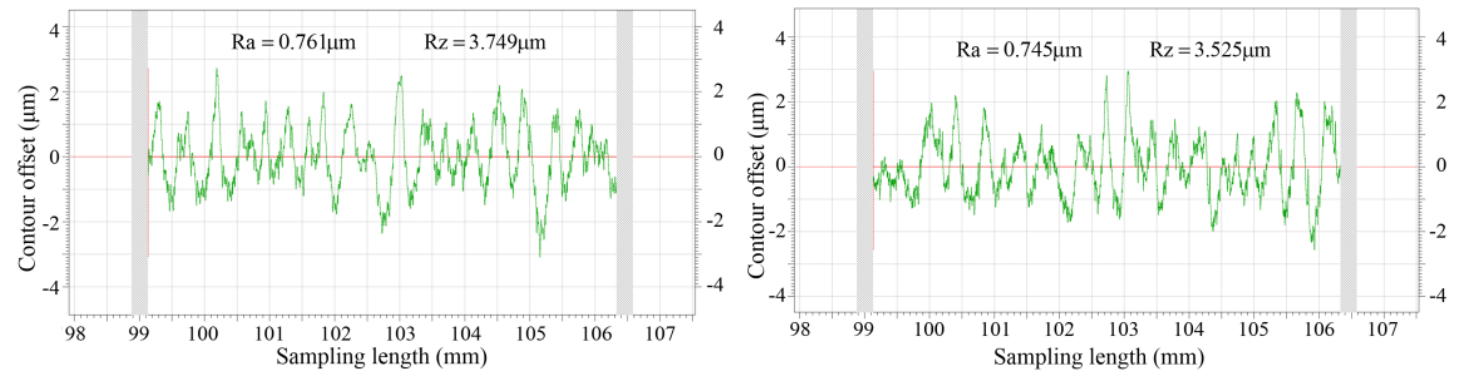

Fig. 28 Surface roughness in direction $B$ before and after optimizing for tool axis vector.

In order to better reflect the surface quality after processing, the Alicona three-dimensional shape measuring instrument is used to scan and measure the position with large curvature on the curved surface, as shown in Fig. 26. The cross-sectional analysis is performed on the scan area to obtain the crosssectional profile curves as shown in the Fig. 29. In order to compare the measured cross-sectional profiles, the measured data points are fitted in MATLAB to obtain the contours after surface machining, as shown in Fig. 30. The maximum machining error is defined as the difference between the actual machined surface and the ideal surface at the same position, so it can be seen from the figure that the contour of the machined surface which tool axis vectors are optimized is closer to the ideal surface. And as shown in the Fig. 30, the maximum machining error is reduced from $0.7 \mathrm{~mm}$ to $0.2 \mathrm{~mm}$ after the tool axis vector optimization, which is reduced by $70 \%$.

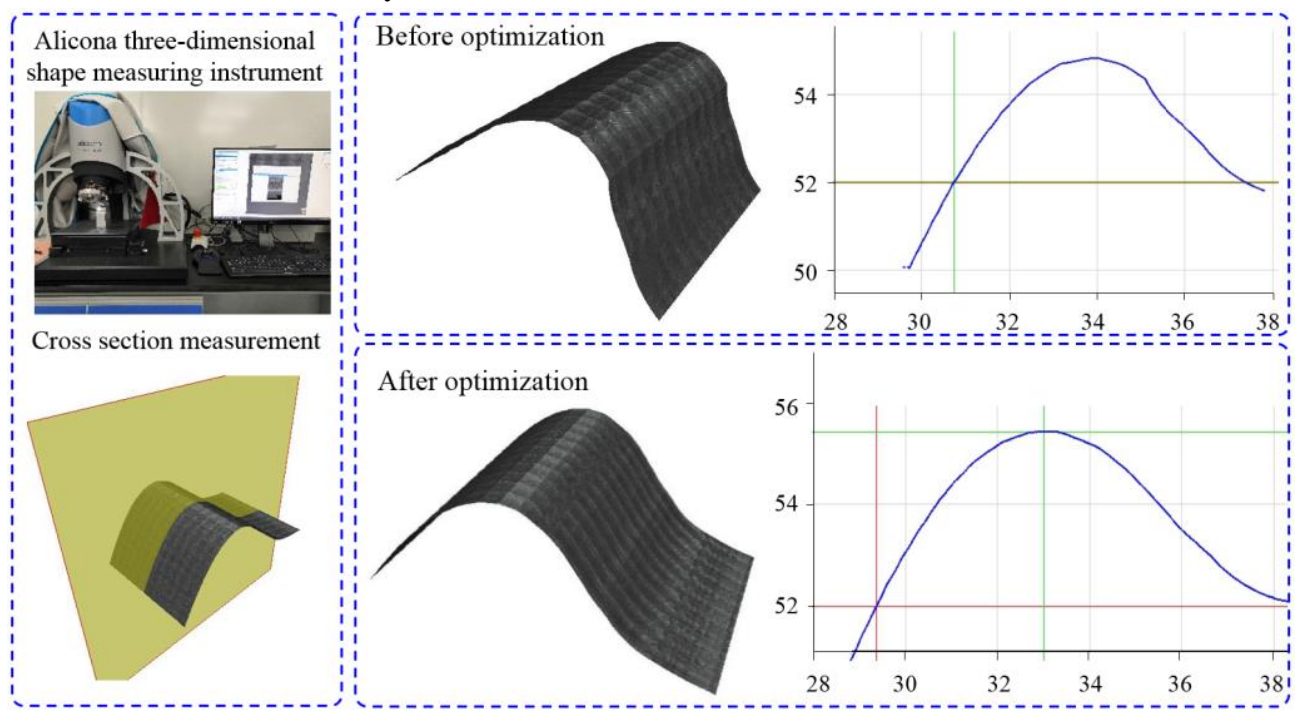

Fig. 29 Scanning section and contour before and after tool axis vector optimization. 


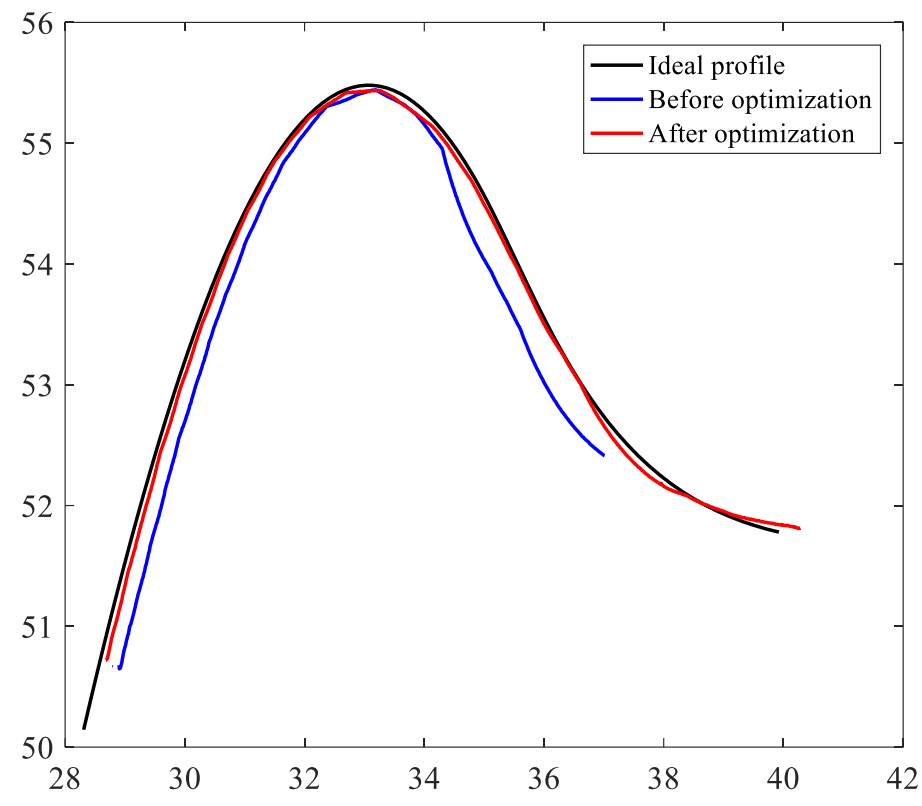

Fig. 30 Surface contour fitting curve.

\section{Conclusions}

In the 5-axis NC machining process of complex curved surface, affected by the shape of the surface, the local optimization method of tool axis vector may not reduce the angular velocity and angular acceleration of rotary feed axis because the optimization intervals are too much. In order to solve this issue effectively, the global optimization method of tool axis vectors is proposed for curved surface machining in this paper. Without changing the cutter contact points at the machined surface, the angular velocity and the angular acceleration of the rotary feed axis can be reduced by tool axis vector optimization method aiming at minimum angular acceleration, which can improve the machining quality of complex curved surface with sharp curvature. Firstly, the feasible spaces of tool axis vectors at the cutter contact points are obtained. And the toolpath is divided by the concavity and convexity of the toolpath curve, and the key tool axis vector on the toolpath curve is determined. Then, the tool axis vectors are optimized based on the minimum angular acceleration in each interval, and the tool axis vectors at the joint position of the adjacent segment are adjusted to avoid the abrupt change of tool axis vectors. Finally, simulation and experiment results verify that the global optimization method of tool axis vectors proposed in this study can effectively reduce the kinematical parameters for the rotary feed axis of machine tools, improve the quality of the machined surface and reduce machining errors. In summary, the research results in this paper can significantly improve the surface machining quality, and provide a guarantee for high-quality and efficient surface processing.

\section{Acknowledgements}

The project is supported by National Science and Technology Major Project of China (No. 2016ZX04001-002), National Natural Science Foundation of China (No. 51675081), Science Challenge Project of China (No. JCKY2016212A506-0201), Science and Technology Innovation Fund of Dalian (No. 2018J12GX038), Innovation Project for Supporting High-level Talent in Dalian (No. 2016RQ012), Science Fund for Creative Research Groups (No. 51621064) and the Fundamental Research Funds for the Central Universities. The authors wish to thank the anonymous reviewers for their comments which led to improvements of this paper.

\section{References}


[1] Ahmed A, Chen ZZC (2017) A comprehensive approach to determining minimum cutter lengths for five-axis milling. International Journal of Advanced Manufacturing Technology 90(5-8): 1235 1252.

[2] Li WL, Wang G, Zhang G, Li QD, Yin ZP (2017) Interference-free inspection path generation for impeller blades using an on-machine probe. IEEE/ASME Transactions on Mechatronics 22(3): 1218-1226.

[3] Tang TD, Bohez ELJ (2015) A new collision avoidance strategy and its integration with collision detection for five-axis NC machining. International Journal of Advanced Manufacturing Technology 81(5-8): 1247-1258.

[4] Morishige K, Kase K, Takeuchi Y (1997) Collision-free tool path generation using 5-dimensional C-space for 5-axis control machining. International Journal of Advanced Manufacturing Technology 13(6): 393-400.

[5] Jung JY, Ahluwalia RS (2005) NC toolpath generation for 5-axis machining of free formed surfaces. Journal of Intelligent Manufacturing 16(1): 115-127.

[6] Wang J, Luo M, Zhang DH (2018) A GPU-accelerated approach for collision detection and tool posture modification in multi-axis machining. IEEE Access 6: 35132-35142.

[7] Wang GX, Shu QL, Wang J (2014) Research on interference inspection of five-axis machining of complex surfaces. China Mechanical Engineering 25(3):299-303.

[8] Sun Y, Lin H (2011) Spiral tool-path generation with constant scallop height for high-speed machining. Journal of Computer-Aided Design \& Computer Graphics 23(7):1249-1253.

[9] Chiou CJ , Lee YS (2002) A machining potential field approach to toolpath generation for multiaxis sculptured surface machining. Computer-Aided Design 34(5):357-371.

[10] Srijuntongsiri G, Makhanov SS (2015) Optimisation of five-axis machining G-codes in the angular space. International Journal of Production Research 53(11): 3207-3227.

[11] Tang QC, Yin SH, Zhang Y, Wu JZ (2018) A tool vector control for laser additive manufacturing in five-axis configuration. International Journal of Advanced Manufacturing Technology 98(58):1671-1684.

[12] Tunc LT, Budak E, Bilgen S, Zatarain M (2016) Process simulation integrated tool axis selection for 5-axis tool path generation. CIRP Annals-Manufacturing Technology 65(1): 381-384.

[13] Zhu Y, Chen ZT, Ning T, Xu RF (2016) Tool orientation optimization for 3+2-axis CNC machining of sculptured surface. Computer-Aided Design 77: 60-72.

[14] Lin ZW, Fu JZ, Yao XH, Sun YF (2015) Improving machined surface textures in avoiding fiveaxis singularities considering tool orientation angle changes. International Journal of Machine Tools and Manufacture 98: 41-49.

[15] Xu RF, Chen ZT, Chen WY (2011) Tool positioning algorithm based on smooth toolpaths for 5axis machining of sculptured surfaces. Chinese Journal of Mechanical Engineering 24(5): 851-858.

[16] Liu HJ, Cao NJ, Zhao JB (2015) Research on tool axis vector optimization based on graph. China Mechanical Engineering 26(19): 2629-2632.

[17] Ma JW, Hu GQ, Qin FZ, Su WW, Jia ZY (2019) Optimization method of tool axis vector based on kinematical characteristics of rotary feed axis for curved surface machining. International Journal of Advanced Manufacturing Technology 100(5-8): 2007-2020.

[18] Zhou B, Zhao JB, Liu WJ (2013) Method study on optimization of tool axis vector for complex surface of 5-axis CNC. Journal of Mechanical Engineering 49(7): 184-192. 
[19] Jun CS, Cha K, Lee YS (2003) Optimizing tool orientations for 5-axis machining by configuration space search method. Computer-Aided Design 35(6): 549-566.

[20] Wang N, Tang K (2007) Automatic generation of gouge-free and angular-velocity-compliant fiveaxis toolpath. Computer-Aided Design 39(10): 849-852.

[21] Ho MC, Hwang YR, Hu CH (2003) Five-axis tool orientation smoothing using quaternion interpolation algorithm. International Journal of Machine Tools and Manufacture 43(12): 12591267.

[22] Hu PC, Chen LF, Tang K (2017) Efficiency-optimal iso-planar tool path generation for five-axis finishing machining of freeform surfaces. Computer-Aided Design 83: 33-50.

[23] Tunc LT, Budak E, Bilgen S, Zatarain M (2016) Process simulation integrated tool axis selection for 5-axis tool path generation. CIRP Annals-Manufacturing Technology 65(1): 381-384. 